\title{
HÖLDER REGULARITY AND EXPONENTIAL DECAY OF CORRELATIONS FOR A CLASS OF PIECEWISE PARTIALLY HYPERBOLIC MAPS.
}

\author{
RAFAEL A. BILBAO, RICARDO BIONI, AND RAFAEL LUCENA \\ ABstract. We consider a class of endomorphisms which contains a set \\ of piecewise partially hyperbolic skew-products with a non-uniformly \\ expanding base map. The aimed transformation preserves a foliation \\ which is almost everywhere uniformly contracted with possible disconti- \\ nuity sets, which are parallel to the contracting direction. We prove that \\ the associated transfer operator, acting on suitable anisotropic normed \\ spaces, has a spectral gap (on which we have quantitative estimation) \\ and the disintegration of the unique invariant physical measure, along \\ the stable leaves, is $\zeta$-Hölder. We use this fact to obtain exponential \\ decay of correlations on the set of $\zeta$-Hölder functions.
}

\section{INTRODUCTION}

In this paper, we take advantage of the new ideas on the construction of anisotropic spaces, introduced by [21], to study the behaviour of the transfer operator associated to maps $F: \Sigma \longrightarrow \Sigma$ which has partially hyperbolic skew product structure with a non-uniformly expanding quotient map $f$ : $M \longrightarrow M$. In other words, we prove spectral gap for the transfer operator associated to $F$ and explore some consequences. For instance, we prove that this sort of system has a physical measure which admits a Hölder regular disintegration along the stable foliation. Here, this is enough to have some other statistical properties like exponential decay of correlations on the set of $\zeta$-Hölder functions.

Usually, this sort of study is carried out as an application of the IonescuTulcea and Marinescu's Theorem, by constructing a pair of suitable spaces of functions, a stronger and an auxiliary weaker space, such that the action of the Perron-Frobenius operator on the stronger space has spectral gap (see [6], 32, [10, 19] and [38] for some introductory texts).

In recent years, the technique of finding good anisotropic norms was extended to piecewise hyperbolic systems (see e.g. [8, 77, 9], [16], 24] and [5], [15] for recent papers containing a survey of the topic). From these properties, several limit theorems or stability statements can be deduced. In these approaches, the existence of an expanding direction, enough regularity of the system or transversality between the map's singular set and the

Date: June 3, 2020.

Key words and phrases. Spectral Gap, Statistical Properties, Transfer Operator. 
contracting directions is required, which make them not suitable for our situation.

Despite not giving explicit bounds on the rate of decay of correlations, the application of the ITM's Theorem has shown to be fruitful, especially for the study of piecewise expanding maps (see [36], for instance). Alternatively, [31 took advantage of Garrett Birkhoff's idea, and by defining a Hilbert metric on a cone of functions restricted to which the $\mathbf{P F}$ operator maps the cone strictly inside itself, explicit bounds on the rate of decay of correlations was obtained for a class of non-Markov piecewise expanding systems on the interval.

The Hilbert metric's idea has shown to be powerful and became quite standard. Moreover, its application bypasses the boundary of the expanding maps. An important application of this technique was given in [37] and [14] in the analysis of the spectrum of the Ruelle-Perron-Frobenius operator, in order to study the Thermodynamic Formalism for a sort of mostly expanding maps. They prove, among other results, statements on exponential decay of correlations, central limit theorems and stability. Another application of the projective metric's technique was given in [13. That work deals with diffeomorphisms onto its image and proves exponential decay of correlations for Hölder continuous observables and central limit theorem for the maximal entropy probability measure.

In this sense, since the maps presented here are not too regular in horizontal direction and maybe not invertible, the results reached in the present work generalize the ones given in [13. Here, we deal with endomorphisms $F$ with discontinuity sets (if non-empty) parallel to the stable direction. We study the action of the transfer operator of $F: M \times K \longrightarrow M \times K$ on a suitable normed space of signed measures. These vector spaces are constructed by identifying a measure on the square, $M \times K$, with a path of measures $M \longmapsto S M(K)(S M(K)$ denotes the space of signed measures on $K)$, where $S M(K)$ is endowed with the "dual of Hölder" norm. This is achieved by generalizing the Wasserstein-Kantorovich-like norm defined in [21] (see also 28. for similar applications of the Wasserstein distance to obtain limit theorems) and this is enough to obtain the limit theorems on a larger Banach space of functions as an application of Theorem $\mathrm{D}$, where we prove that the disintegration of the physical $F$-invariant measure is $\zeta$-Hölder regular. This sort of result has many other applications. For example, the reader can see [21] and [20], where a stability result was proved for Lorenz-like systems and partially hyperbolic skew products, respectively, under ad-hoc perturbations of the system.

Statements of the Main Results. Here we expose the main results of this work. We note that, the hypothesis (f1), (f2), (f3) and (G1) on the system $F$ will be stated in section 2 and (G2) in section 7 .

The first result guaranties existence and uniqueness for the $F$-invariant measure in the space $S^{\infty}$ (see equation (15)). Its proof is given in section 5.1 . 
Theorem A. There exists a unique F-invariant probability, $\mu_{0} \in S^{\infty}$. Moreover, $\pi_{x *} \mu_{0}=m_{1}$.

Next result, where the proof can be found in section [6. shows that the transfer operator acting on the space $S^{\infty}$ is quasi-compact. This sort of result has many consequences for the dynamic and it implies several limit theorems. For instance, we obtain an exponential rate of convergence for the limit

where

$$
\lim _{n \rightarrow \infty} C_{n}(f, g)=0
$$

$$
C_{n}(f, g):=\left|\int\left(g \circ F^{n}\right) f d \mu_{0}-\int g d \mu_{0} \int f d \mu_{0}\right|,
$$

$g: M \times K \longrightarrow \mathbb{R}$ is a $\zeta$-Holder function and $f \in \Theta_{\mu_{0}}$. The set $\Theta_{\mu_{0}}$ is defined as

$$
\Theta_{\mu_{0}}:=\left\{f: M \times K \longrightarrow \mathbb{R} ; f \mu_{0} \in S^{\infty}\right\},
$$

where the measure $f \mu_{0}$ is defined by $f \mu_{0}(E):=\int_{E} f d \mu_{0}$, for all measurable set $E$.

Theorem B (Spectral gap on $S^{\infty}$ ). If $F: \Sigma \longrightarrow \Sigma$ satisfies (f1), (f2), (f3) and (G1) given at beginning of section [2.1, then the operator $\mathrm{F}_{*}: S^{\infty} \longrightarrow$ $S^{\infty}$ can be written as

$$
\mathrm{F}_{*}=\mathrm{P}+\mathrm{N}
$$

where

a) $\mathrm{P}$ is a projection, i.e., $\mathrm{P}^{2}=\mathrm{P}$ and $\operatorname{dim} \mathrm{P}\left(S^{\infty}\right)=1$;

b) there are $0<\xi<1$ and $K>0$ such that $\forall \mu \in S^{\infty}$

$$
\left\|\mathrm{N}^{n}(\mu)\right\|_{S^{\infty}} \leq\|\mu\|_{S^{\infty}} \xi^{n} K
$$

c) $\mathrm{PN}=\mathrm{NP}=0$.

Next proposition is a consequence of all previous theorems. It shows that the system $F$ has exponential decay of correlations $\left(\lim _{n \rightarrow \infty} C_{n}(f, g)=0\right.$ exponentially fast) for observables $f \in \Theta_{\mu_{0}}$ and $g \in \mathrm{H}_{\zeta}(\Sigma)$. Its proof is given in section 6.1,

Theorem C. For every $\zeta$-Hölder function $g: \Sigma \longrightarrow \mathbb{R}$ and all $f \in \Theta_{\mu_{0}}$, it holds

$$
\left|\int\left(g \circ F^{n}\right) f d \mu_{0}-\int g d \mu_{0} \int f d \mu_{0}\right| \leq|| f \mu_{0} \|_{S^{\infty}} K|g|_{\zeta} \xi^{n} \quad \forall n \geq 1,
$$

where $\xi$ and $K$ are from Theorem $B$ and $|g|_{\zeta}:=|g|_{\infty}+H_{\zeta}(g)$.

The following theorem is an estimate for the Holder's constant (see equation (32) in Definition (7.3) of the disintegration of the unique $F$-invariant measure $\mu_{0}$ in $S^{\infty}$. This kind of result has many applications and similar estimations (for other systems) were given in [21] and [12]. In [21], for instance, they use the regularity of the disintegration to prove stability of 
the $F$-invariant measure under a kind of $a d$-hoc perturbation. Here, we use this result to show that the abstract set $\Theta_{\mu_{0}}$, defined above, contains the $\zeta$-Holders functions. The proof of the following result is presented in section 7.

Theorem D. Suppose that $F: \Sigma \longrightarrow \Sigma$ satisfies (f1), (f2), (f3), (G1), (G2) and $(\alpha \cdot L)^{\zeta}<1$ and consider the unique $F$-invariant probability $\mu_{0} \in S^{\infty}$. Then $\mu_{0} \in \mathcal{H}_{\zeta}^{+}$and

$$
\left|\mu_{0}\right| \leq \frac{D}{1-\beta}
$$

where $D$ and $\beta$ are from Proposition 7.7 .

As a consequence of the estimative given in the previous theorem, next theorem complements the Theorem C. It implies, for instance,

$$
\lim _{n \rightarrow \infty} C_{n}(f, g)=0
$$

exponentially fast for all Holder observable $f, g \in \mathrm{H}_{\zeta}(\Sigma)$. Its demonstration is given in section 8 .

Theorem E. Suppose that $F: \Sigma \longrightarrow \Sigma$ satisfies (f1), (f2), (f3), (G1), (G2) and $(\alpha \cdot L)^{\zeta}<1$ and let $\mu_{0}$ be the unique $F$-invariant measure in $S^{\infty}$. Then, $\mathrm{H}_{\zeta}(\Sigma) \subset \Theta_{\mu_{0}}$.

Plan of the paper. The paper is structured as follows:

- Section 2: we introduce the kind of systems we consider in the paper. Essentially, it is a class of systems which contains a set of piecewise partially hyperbolic dynamics $(F(x, y)=(f(x), G(x, y)))$ with a non-uniformly expanding basis map, $f$, and whose fibers are uniformly contracted $m_{1}$-a.e, where $m_{1}$ is an $f$-invariant probability measure. Here and until section 7, where more regularity is required, we do not ask for any kind of regularity on $G$ in the horizontal direction (for the functions $x \longmapsto G(x, y), y$ fixed);

- Section 3: we introduce the functional spaces used in the paper and discussed in the previous paragraphs;

- Section 4: we show the basic properties of the transfer operator of $F$ when applied to these spaces. In particular we see that there is a useful "Perron-Frobenius"-like formula (see Proposition 4.2);

- Section 5: we discuss the basic properties of the iteration of the transfer operator on the spaces we consider. In particular, we prove a Lasota-Yorke inequality and a convergence to equilibrium statement (see Propositions 5.3 and 5.7);

- Section 6: we use the convergence to equilibrium and the LasotaYorke inequalities to prove the spectral gap for the transfer operator associated to the system restricted to a suitable strong space (see Theorem (B) and prove a decay of correlation statement on an abstract set of functions; 
- Section 7: we consider a similar system with some more regularity on the family of functions $\{G(\cdot, y)\}_{y \in K}, x \longmapsto G(x, y)$ : there exists a partition (into open sets) $\mathcal{P}=P_{1}, \cdots, P_{\operatorname{deg}(f)}$, such that the restriction of the function $x \longmapsto G(x, y)$ to $P_{i}$ is $\left(k_{y, i}, \zeta\right)$-Hölder, where the family $\left\{k_{y, i}\right\}_{y \in K}$ is bounded, and it holds for all $i$. This allows discontinuities on the boundaries $\left(\partial P_{i}\right) \times K$. For this sort of system, we prove a stronger regularity result for the iteration of probability measures (see Corollary 7.8 and Remark 7.9) and show that the $F$ invariant physical measure has a $\zeta$-Hölder disintegration along the stable fibers (see Theorem D);

- Section 8: we use the $\zeta$-Hölder regularity of the physical measure established in section 7 , to prove that the abstract set of functions on which the system has decay of correlations contains all $\zeta$-Hölder functions (see Theorem E).

Acknowledgment We are thankful to Stefano Galatolo for all valuable comments and fruitful discussions regarding this work.

This work was partially supported by Alagoas Research Foundation-FAPEAL (Brazil) Grants 60030 000587/2016, CNPq (Brazil) Grants 300398/2016-6, CAPES (Brazil) Grants 99999.014021/2013-07 and EU Marie-Curie IRSES Brazilian-European partnership in Dynamical Systems (FP7-PEOPLE- 2012IRSES 318999 BREUDS).

\section{Settings}

Fix a compact and connected Riemannian manifold, $M$, equipped with its Riemannian metric $d_{1}$. For the sake of simplicity, we suppose that $\operatorname{diam}(M)=1$, this is not restrictive but will avoid multiplicative constants. Moreover, consider a compact metric space $\left(K, d_{2}\right)$, endowed with its Borel's sigma algebra, $\mathcal{B}$. We set $\Sigma:=M \times K$ and we endow this space with the metric $d_{1}+d_{2}$.

2.1. Contracting Fiber Maps with Non Uniformily Expanding Basis. Let $F$ be the map $F: \Sigma \longrightarrow \Sigma$ given by

$$
F(x, z)=(f(x), G(x, z)),
$$

where $G: \Sigma \longrightarrow K$ and $f: M \longrightarrow M$ are measurable maps satisfying what follows.

2.1.1. Hypothesis on $f$. Suppose that $f: M \longrightarrow M$ is a local diffeomorphism and assume that there is a continuous function $L: M \longrightarrow \mathbb{R}$, s.t. for every $x \in M$ there exists a neighbourhood $U_{x}$, of $x$, so that $f_{x}:=\left.f\right|_{U_{x}}: U_{x} \longrightarrow$ $f\left(U_{x}\right)$ is invertible and

$$
d_{1}\left(f_{x}^{-1}(y), f_{x}^{-1}(z)\right) \leq L(x) d_{1}(y, z), \quad \forall y, z \in f\left(U_{x}\right) .
$$

In particular, $\# f^{-1}(x)$ is constant for all $x \in M$. We set $\operatorname{deg}(f):=$ $\# f^{-1}(x)$, the degree of $f$. 
Denote by

$$
\rho(\gamma):=\frac{1}{\left|\operatorname{det}\left(f^{\prime}(\gamma)\right)\right|},
$$

where $\operatorname{det}\left(f^{\prime}\right)$ is the Jacobian of $f$ with respect to $m_{1}$.

Suppose that there is an open region $\mathcal{A} \subset M$ and constants $\sigma>1$ and $L \geq 1$ such that

(f1) $L(x) \leq L$ for every $x \in \mathcal{A}$ and $L(x)<\sigma^{-1}$ for every $x \in \mathcal{A}^{c}$. Moreover, $L$ is close enough to 1 : the precise estimation for $L$ is given in equation (5);

(f2) There exists a finite covering $\mathcal{U}$ of $M$, by open domains of injectivity for $f$, such that $\mathcal{A}$ can be covered by $q<\operatorname{deg}(f)$ of these domains.

Denote by $H_{\zeta}$ the set of the Hölder functions $h: M \longrightarrow \mathbb{R}$, i.e., if we define

$$
H_{\zeta}(h):=\sup _{x \neq y} \frac{|h(x)-h(y)|}{d_{1}(x, y)^{\zeta}}
$$

then

$$
H_{\zeta}:=\left\{h: M \longrightarrow \mathbb{R}: H_{\zeta}(h)<\infty\right\} .
$$

Next, (f3) is an open condition relatively to the Hölder norm and equation (41) means that $\rho$ belongs to a small cone of Hölder continuous functions (see [14]).

(f3) There exists a sufficiently small $\epsilon_{\rho}>0$ s.t.

$$
\sup \log (\rho)-\inf \log (\rho)<\epsilon_{\rho}
$$

and

$$
H_{\zeta}(\rho)<\epsilon_{\rho} \inf \rho .
$$

Precisely, we suppose the constants $\epsilon_{\rho}$ and $L$ satisfy the condition

$$
\exp \epsilon_{\rho} \cdot\left(\frac{(\operatorname{deg}(f)-q) \sigma^{-\alpha}+q L^{\alpha}\left[1+(L-1)^{\alpha}\right]}{\operatorname{deg}(f)}\right)<1 .
$$

According to [14], such a map (satisfying (f1), (f2) and (f3)) $f: M \longrightarrow M$ has an invariant probability $m_{1}$ of maximal entropy, absolutely continuous with respect to a conformal measure, and its Perron-Frobenius operator with respect to $m_{1}, \mathrm{P}_{f}: L_{m_{1}}^{1} \longrightarrow L_{m_{1}}^{1}$, defined for $\varphi \in L_{m_{1}}^{1}$ by $\mathrm{P}_{f}(\varphi)(x)=$ $\sum_{i=1}^{\operatorname{deg}(f)} \varphi\left(x_{i}\right) \rho\left(x_{i}\right)\left(x_{i}\right.$ is the $i$-th pre image of $\left.x, i=1, \cdots, \operatorname{deg}(f)\right)$, satisfies the following result.

Theorem 2.1. There exist $0<r<1$ and $D>0$ s.t. for all $\varphi \in H_{\zeta}$, with $\int \varphi d m_{1}=0$, it holds

$$
\left|\mathrm{P}_{\mathrm{f}}^{n}(\varphi)\right|_{\zeta} \leq D r^{n}|\varphi|_{\zeta} \quad \forall n \geq 1,
$$

where $|\varphi|_{\zeta}:=H_{\zeta}(\varphi)+|\varphi|_{\infty}$ for all $\varphi \in H_{\zeta}$. 
Remark 2.2. By (f2), (see Lemma 2.3) there exists a disjoint finite family, $\mathcal{P}$, of open sets, $P_{1}, \cdots, P_{\operatorname{deg}(f)}$, s.t. $\bigcup_{i=1}^{\operatorname{deg}(f)} P_{i}=M m_{1}$-a.e., and $\left.f\right|_{P_{i}}$ : $P_{i} \longrightarrow f\left(P_{i}\right)$ is a diffeomorfism for all $i=1, \cdots \operatorname{deg}(f)$. Moreover, $f\left(P_{i}\right)=$ $M m_{1}$-a.e., for all $i=1, \cdots, \operatorname{deg}(f)$. Therefore, it holds that

$$
\mathrm{P}_{f}(\varphi)(x)=\sum_{i=1}^{\operatorname{deg}(f)} \varphi\left(x_{i}\right) \rho\left(x_{i}\right) \chi_{f\left(P_{i}\right)}(x),
$$

for $m_{1}$-a.e. $x \in M$, where

$$
\rho_{i}(\gamma):=\frac{1}{\left|\operatorname{det}\left(f_{i}^{\prime}(\gamma)\right)\right|}
$$

and $f_{i}=\left.f\right|_{P_{i}}$. This expression will be used later on.

Lemma 2.3. Let $\left(f, M, m_{1}\right)$ be a non-singular system $\left(m_{1}(A)=0 \Rightarrow\right.$ $m_{1}\left(f^{-1}(A)\right)=0$, where $M$ is a compact and connected manifold and suppose that $m_{1}$ be absolutely continuous with respect to Lebesgue. Suppose that $f: M \rightarrow M$ a local homeomorphism with degree $\operatorname{deg}(f)$. Then, there exist a finite partition $\mathcal{P}=\left\{P_{1}, \cdots, P_{p}\right\}$, where $p=\operatorname{deg}(f), P_{i}$ is open for all $i=1, \cdots, \operatorname{deg}(f)$ and $m_{1}\left(M \backslash f\left(P_{i}\right)\right)=0$ for all $i=1, \cdots, \operatorname{deg}(f)$.

Proof. Since $f$ is a local homeomorphism, $a \in M$, there exists $r_{a}>0$ s.t. $f$ : $V\left(a^{j}\right) \rightarrow B\left(a, r_{a}\right)$ is a homeomorphism for all $j=1, \ldots, p$, where $f^{-1}(a)=$ $\left\{a^{1}, \ldots, a^{p}\right\}$ and $V\left(a^{j}\right) \cap V\left(a^{i}\right)=\emptyset$, for all $j \neq i$. On the other hand, $M=$ $\bigcup_{a \in M} B\left(a, r_{a}\right)$ and by compacity there exist a finite number of balls $B\left(a, r_{a}\right)$ which covers $M$, i.e., $M=\bigcup_{n=1}^{r} B\left(a_{n}, r_{a_{n}}\right)$, where $a_{n} \in M$ for all $n=$ $1, \cdots, r$. From this cover, let $\mathcal{K}$ be a finite partition such that $\# \mathcal{K}=: q$ and the boundary of each atom has $m_{1}$-null measure.

Therefore, for each $K_{t} \in \mathcal{K}$ we have $f^{-1}\left(K_{t}\right)=K_{t}^{1} \cup \ldots \cup K_{t}^{p}$ where $K_{t}^{j} \cap$ $K_{t}^{i}=\emptyset$ for all $j \neq i$ and $1 \leq t \leq q$. Thus, $f: K_{t}^{j} \rightarrow K_{t}$ is a homeomorphism for each $1 \leq t \leq q$ and $1 \leq j \leq p$. Define $M^{\prime}=\bigcup_{K \in \mathcal{K}} \operatorname{int}(K)$ in a way that $m_{1}\left(M \backslash M^{\prime}\right)=0$, where $M \backslash M^{\prime}$ is the boundary of the sets $K \in \mathcal{K}$. Then, define

$$
\begin{gathered}
P^{1}=K_{1}^{1} \cup K_{2}^{1} \cup K_{3}^{1} \cup \cdots \cup K_{q}^{1}, \\
P^{2}=K_{1}^{2} \cup K_{2}^{2} \cup K_{3}^{2} \cup \cdots \cup K_{q}^{2}, \\
\cdot \\
\cdot \\
\cdot \\
P^{p}=K_{1}^{p} \cup K_{2}^{p} \cup K_{3}^{p} \cup \cdots \cup K_{q}^{p} .
\end{gathered}
$$

Finally, we have that $\mathcal{P}:=\left\{P^{j}\right\}_{1 \leq j \leq p}$ is a measurable partition of $M$ and by construction $f: \operatorname{int}\left(P^{j}\right) \rightarrow M^{\prime}$ is a homeomorphism for all $1 \leq j \leq p$. 
2.1.2. Hypothesis on $G$. We suppose that $G: \Sigma \longrightarrow K$ satisfies:

(G1) $G$ is uniformly contracting on $m_{1}$-a.e. vertical fiber, $\gamma_{x}:=\{x\} \times K$. Precisely, there is $0<\alpha<1$ such that for $m_{1}$-a.e. $x \in M$ it holds

$$
d_{2}\left(G\left(x, z_{1}\right), G\left(x, z_{2}\right)\right) \leq \alpha d_{2}\left(z_{1}, z_{2}\right), \quad \forall z_{1}, z_{2} \in K .
$$

We denote the set of all vertical fibers $\gamma_{x}$, by $\mathcal{F}^{s}$ :

$$
\mathcal{F}^{s}:=\left\{\gamma_{x}:=\{x\} \times K ; x \in M\right\} .
$$

When no confusion is possible, the elements of $\mathcal{F}^{s}$ will be denoted simply by $\gamma$, instead of $\gamma_{x}$.

Example 2.1. Let $f_{0}: \mathbb{T}^{d} \longrightarrow \mathbb{T}^{d}$ be a linear expanding map. Fix a covering $\mathcal{P}$ and an atom $P_{1} \in \mathcal{P}$ which contains a periodic point (maybe fixed point) $p$. Then, consider a perturbation $f$, of $f_{0}$, inside $P_{1}$ by a pitchfork bifurcation, in a way that $p$ becomes a saddle for $f$. Therefore, $f$ coincides with $f_{0}$ in $P_{1}^{c}$, where we have uniform expansion. The perturbation can be made in a way that ( $\mathrm{f} 1$ ) is satisfied, i.e., is never too contracting in $P_{1}$ and $f$ is still a topological mixing. Note that a small perturbation with the previous properties may not exist. If it does, then (f3) is satisfied. In this case, $m_{1}$ is absolutely continuous with respect to the Lebesgue measure which is an expanding conformal and positive measure on open sets. Hence, there can be no periodic attractors.

Example 2.2. In the previous example, assume that $f_{0}$ is diagonalisable, with eigenvalues $1<1+a<\lambda$, associated to $e_{1}, e_{2}$ respectively, and $x_{0}$ is a fixed point. Fix $a, \epsilon>0$ such that $\log \left(\frac{1+a}{1-a}\right)<\epsilon$ and

$$
\exp \epsilon\left(\frac{\left(\operatorname{deg}\left(f_{0}\right)-1\right)(1+a)^{-\alpha}+(1 /(1-a))^{\alpha}\left[1+(a /(1-a))^{\alpha}\right]}{\operatorname{deg}\left(f_{0}\right)}\right)<1 .
$$

Note that any smaller $a>0$ will still satisfy these equations.

Let $\mathcal{U}$ be a finite covering of $M$ by open domains of injectivity for $f$. Redefining sets in $\mathcal{U}$, we may assume $x_{0}=\left(m_{0}, n_{0}\right)$ belongs to exactly one such domain $U$. Let $r>0$ be small enough that $B_{2 r}\left(x_{0}\right) \subset U$. Define $\rho=\eta_{r} * g$, where $\eta_{r}(z)=\left(1 / r^{2}\right) \eta(z / r), \eta$ the standard mollifier, and

$$
g(m, n)= \begin{cases}\lambda(1-a), & \text { if }(m, n) \in B_{r}\left(x_{0}\right) \\ \lambda(1+a), & \text { otherwise. }\end{cases}
$$

Finally, define a perturbation $f$ of $f_{0}$ by

$$
f(m, n)=\left(m_{0}+\lambda\left(m-m_{0}\right), n_{0}+(\rho(m, n) / \lambda)\left(n-n_{0}\right)\right) .
$$

Then $x_{0}$ is a saddle point of $f$ and the desired conditions are satisfied for $\mathcal{A}=B_{2 r}\left(x_{0}\right), L=1 /(1-a)$ and $\sigma=1+2 a$. The only non-trivial condition is (f3). To show it, note that

$$
\rho(x)-\rho(y)=\int_{S} \frac{2 a}{\lambda\left(1-a^{2}\right)} \eta_{r}(z) d z-\int_{S^{\prime}} \frac{2 a}{\lambda\left(1-a^{2}\right)} \eta_{r}(z) d z,
$$


where $S=\left\{z \in \mathbb{R}^{2}: x-z \in B_{r}\left(x_{0}\right), y-z \notin B_{r}\left(x_{0}\right)\right\}$ and $S^{\prime}=\left\{z \in \mathbb{R}^{2}\right.$ : $\left.y-z \in B_{r}\left(x_{0}\right), x-z \notin B_{r}\left(x_{0}\right)\right\}$. Take $x, y \in \mathbb{R}^{2}$ and write $|x-y|=q r$, $A_{q}=\left\{z \in \mathbb{R}^{2}: 1-q<|z|<1\right\}$. We have

$$
\frac{|\rho(x)-\rho(y)|}{|x-y|^{\zeta}} \leq \frac{2 a \eta_{r}(S)}{\lambda\left(1-a^{2}\right) q^{\zeta} r^{\zeta}} \leq \frac{2 a \eta\left(A_{q}\right) / q^{\zeta}}{\lambda\left(1-a^{2}\right)}
$$

Since $N=\sup _{q>0} \eta\left(A_{q}\right) / q^{\zeta}<+\infty$, we can take $a$ so small that $2 a N /(1-$ a) $<\epsilon$, therefore $H_{\zeta}(\rho)<\epsilon \inf \rho$.

2.2. A Lasota-Yorke Inequality for $\mathrm{P}_{f}$. In this section, we provide a Lasota-Yorke inequality for the Perron-Frobenius Operator of $f$, acting on the space of Hölder functions. The proof follows from a general approach, where such a linear operator has spectral gap.

Lemma 2.4. Let $\left(B_{w},\|\cdot\|_{w}\right)$ and $\left(B_{s},\|\cdot\|_{s}\right)$ be two Banach spaces, with $B_{s} \subset B_{w}$ and let $L: B_{w} \rightarrow B_{w}$ be a bounded linear operator s.t. $L\left(B_{s}\right) \subset B_{s}$. Suppose there exists a projection $P: B_{w} \rightarrow B_{w}^{1} \subset B_{s}$, bounded with respect to the norms $\|\cdot\|_{w}$ and $\|\cdot\|_{B_{w} \rightarrow B_{s}}$, such that $L\left(B_{w}^{1}\right) \subset B_{w}^{1}$.

If there exist $q<1$ and $Q \in \mathbb{R}$ such that

$$
\forall n \in \mathbb{N}:\left\|\left.L^{n}\right|_{B_{s} \cap B_{w}^{0}}\right\|_{s} \leq Q q^{n}
$$

where $B_{w}^{0}:=P^{-1}(0)$, then there exists $N \in \mathbb{N}, r<1$ and $R \in \mathbb{R}$ s.t.

$$
\left\|L^{N} g\right\|_{s} \leq r\|g\|_{s}+R\|g\|_{w}
$$

for all $g \in B_{s}$.

Proof. For each $g \in B_{s}$ and $n \in \mathbb{N}$ we have that

$$
\left\|L^{n}(g-P g)\right\|_{s} \leq Q q^{n}\|g-P g\|_{s}
$$

by the triangle inequality,

$$
\begin{gathered}
\|g-P g\|_{s} \leq\|g\|_{s}+\|P g\|_{s} \\
\left\|L^{n} g\right\|_{s} \leq\left\|L^{n}(g-P g)\right\|_{s}+\left\|L^{n} P g\right\|_{s} .
\end{gathered}
$$

Since $L\left(B_{w}^{1}\right) \subset B_{w}^{1}$, we get that

$$
\left\|L^{n} g\right\|_{s} \leq Q q^{n}\left(\|g\|_{s}+\|P g\|_{s}\right)+\left\|P L^{n} P g\right\|_{s} .
$$

Letting $n=N \in \mathbb{N}$ large enough so that $Q q^{N}<1$, we obtain (8) with $r=Q q^{N}$ and $R=\|P\|_{B_{w} \rightarrow B_{s}}\left(Q q^{N}+\left\|L^{N}\right\|_{w}\|P\|_{w}\right)$.

Lemma 2.5. Let $\left(B_{w},\|\cdot\|_{w}\right)$ and $\left(B_{s},\|\cdot\|_{s}\right)$ be Banach spaces, with $B_{s} \subset B_{w}$ where $\iota: B_{s} \rightarrow B_{w}$ is bounded and let $L: B_{w} \rightarrow B_{w}$ be a bounded linear operator s.t. $L\left(B_{s}\right) \subset B_{s}$ and $\sup _{n \in \mathbb{N}}\left\|L^{n}\right\|_{w}<\infty$. Assume that there exists a decomposition $B_{w}=B_{w}^{0} \oplus B_{w}^{1}$ into invariant subspaces $B_{w}^{0}$ and $B_{w}^{1}$ such that the inequality

$$
\forall n \in \mathbb{N}:\left\|\left.L^{n}\right|_{B_{s} \cap B_{w}^{0}}\right\|_{s} \leq Q q^{n}
$$


holds for some $q<1$ and $Q \in \mathbb{R}$. Suppose that $\left.L\right|_{B_{w}^{1}}$ is diagonalizable with unit spectrum, $B_{w}^{1} \subset B_{s}$ and $\operatorname{dim} B_{w}^{1}<\infty$. Then there exist $N \in \mathbb{N}, r<1$ and $R \in \mathbb{R}$ such that

$$
\left\|L^{N} g\right\|_{s} \leq r\|g\|_{s}+R\|g\|_{w}
$$

for all $g \in B_{s}$.

Proof. Take a basis of eigenvectors $\left\{h_{1}, \ldots, h_{d}\right\}$ of $B_{w}^{1}$ and consider the linear isomorphism

$$
\begin{aligned}
A: B_{w}^{1} & \rightarrow \mathbb{R}^{d} \\
\sum_{i=1}^{d} \alpha_{i} h_{i} & \mapsto\left(\alpha_{1}, \ldots, \alpha_{d}\right) .
\end{aligned}
$$

Denote by $\|\cdot\|_{2}$ the Euclidian norm in $\mathbb{R}^{d}$. Since $\left.L\right|_{B_{w}^{1}}$, has unit spectrum and $\left\{h_{1}, \ldots, h_{d}\right\}$ is a basis of eigenvectors, we have that

$$
\forall n \in \mathbb{N}:\left\|A L^{n} g^{1}\right\|_{2}=\left\|A g^{1}\right\|_{2}
$$

for all $g^{1} \in B_{w}^{1}$. Therefore, for any $n \in \mathbb{N}, g^{1} \in B_{w}^{1}$ and $i, j \in\{w, s\}$,

$$
\frac{1}{M_{i, j}} \leq \frac{\left\|L^{n} g^{1}\right\|_{i}}{\left\|g^{1}\right\|_{j}} \leq M_{i, j}:=\left\|A^{-1}\right\|_{i, 2}\|A\|_{2, j} \text {. }
$$

Thus, the projection $P: B_{w} \rightarrow B_{w}^{1}$ satisfies

$$
\begin{aligned}
\|P g\|_{s} & \leq\left\|L^{n} P g\right\|_{w} M_{w, s} \\
& \leq\left\|L^{n} g\right\|_{w} M_{w, s}+\left\|L^{n}(g-P g)\right\|_{w} M_{w, s} \\
& \leq \sup _{n \in \mathbb{N}}\left\|L^{n}\right\|_{w}\|g\|_{w} M_{w, s}+\|\iota\|_{+, *}\left\|L^{n}(g-P g)\right\|_{s} M_{w, s} \\
& \leq \sup _{n \in \mathbb{N}}\left\|L^{n}\right\|\|g\|_{w} M_{w, s}+\|\iota\|_{+, *} Q q^{n}\|g\|_{s} M_{w, s} .
\end{aligned}
$$

Letting $n \rightarrow+\infty$,

$$
\|P g\|_{s} \leq \sup _{n \in \mathbb{N}}\left\|L^{n}\right\|\|g\|_{w} M_{w, s} .
$$

Thus, the projections $P$ is $\left(B_{w}, B_{s}\right)$-bounded and so $B_{w}$-bounded since $\iota$ is continuous. Hence, the hypothesis of Lemma 2.4 are satisfied.

Theorem 2.6. (Lasota-Yorke inequality) There exist $k \in \mathbb{N}, 0<\beta_{0}<1$ and $C>0$ such that, for all $g \in H_{\zeta}$, it holds

$$
\left|\mathrm{P}_{f}^{k} g\right|_{\zeta} \leq \beta_{0}|g|_{\zeta}+C|g|_{\infty}
$$

where $|g|_{\zeta}:=H_{\zeta}(g)+|g|_{\infty}$.

Proof. Let $\left(B_{w},\|\cdot\|_{w}\right)=\left(C_{0},|\cdot|_{\infty}\right),\left(B_{s},\|\cdot\|_{s}\right)=\left(H_{\zeta},|\cdot|_{\zeta}\right), L=\mathrm{P}_{f}$, and $f(\varphi)=\int \varphi d m_{1}$. The inclusion $\iota: B_{s} \rightarrow B_{w}$ is bounded and $L: B_{w} \rightarrow B_{w}$ is a bounded linear operator such that $L\left(B_{s}\right) \subset B_{s} . h=1$ satisfies $L h=h \neq 0$, $f: B_{w} \rightarrow \mathbb{R}$ is bounded and $f^{-1}(0)=\left\{\varphi \in H_{0}: \int \varphi d m_{1}=0\right\}$. Take $B_{w}^{0}=f^{-1}(0)$ and $B_{w}^{1}=\mathbb{R} h$ in Lemma 2.5. Since Theorem 2.1 implies condition (9), the hypothesis of the Lemma are satisfied. 
The following is a standard consequence of Theorem 2.6 (and the fact that 1 is a fixed point for $\mathrm{P}_{f}$ ) that allows us to estimate the behaviour of any given power of the transfer operator.

Corollary 2.7. There exist constants $B_{3}>0, C_{2}>0$ and $0<\beta_{2}<1$ such that for all $g \in H_{\zeta}$, and all $n \geq 1$, it holds

$$
\left|\mathrm{P}_{f}^{n} g\right|_{\zeta} \leq B_{3} \beta_{2}^{n}|g|_{\zeta}+C_{2}|g|_{\infty},
$$

where $|g|_{\zeta}:=H_{\zeta}(g)+|g|_{\infty}$.

\section{WEAK AND STRONG SPACES}

3.1. $L^{\infty}$-like spaces. Through this section, we construct some function spaces which are suitable for the systems defined in section 2.1. The idea is to define spaces of signed measures, where the norms are provided by disintegrating measures along the stable foliation. Thus, a signed measure will be seen as a family of measures on each leaf. For instance, a measure on the square with a vertical foliation will be seen as a one parameter family (a path) of measures on the interval (a stable leaf), where this identification will be done by means of the Rokhlin's Disintegration Theorem. Finally, in the vertical direction (on the leaves), we will consider a norm which is the dual of the $\zeta$-Hölder norm and in the "horizontal" direction we will consider essentially the $L_{m_{1}}^{\infty}$ norm.

Rokhlin's Disintegration Theorem. Now we briefly recall disintegration of measures.

Consider a probability space $(\Sigma, \mathcal{B}, \mu)$ and a partition $\Gamma$ of $\Sigma$ into measurable sets $\gamma \in \mathcal{B}$. Denote by $\pi: \Sigma \longrightarrow \Gamma$ the projection that associates to each point $x \in M$ the element $\gamma_{x}$ of $\Gamma$ which contains $x$, i.e., $\pi(x)=\gamma_{x}$. Let $\widehat{\mathcal{B}}$ be the $\sigma$-algebra of $\Gamma$ provided by $\pi$. Precisely, a subset $\mathcal{Q} \subset \Gamma$ is measurable if, and only if, $\pi^{-1}(\mathcal{Q}) \in \mathcal{B}$. We define the quotient measure $\mu_{x}$ on $\Gamma$ by $\mu_{x}(\mathcal{Q})=\mu\left(\pi^{-1}(\mathcal{Q})\right)$.

The proof of the following theorem can be found in [35], Theorem 5.1.11 (items a), b) and c)) and Proposition 5.1.7 (item d)).

Theorem 3.1. (Rokhlin's Disintegration Theorem) Suppose that $\Sigma$ is a complete and separable metric space, $\Gamma$ is a measurable partition of $\Sigma$ and $\mu$ is a probability on $\Sigma$. Then, $\mu$ admits a disintegration relative to $\Gamma$, i.e., a family $\left\{\mu_{\gamma}\right\}_{\gamma \in \Gamma}$ of probabilities on $\Sigma$ and a quotient measure $\mu_{x}$ as above, such that:

(a) $\mu_{\gamma}(\gamma)=1$ for $\mu_{x}$-a.e. $\gamma \in \Gamma$;

(b) for all measurable set $E \subset \Sigma$ the function $\Gamma \longrightarrow \mathbb{R}$ defined by $\gamma \longmapsto$ $\mu_{\gamma}(E)$, is measurable;

(c) for all measurable set $E \subset \Sigma$, it holds $\mu(E)=\int \mu_{\gamma}(E) d \mu_{x}(\gamma)$.

(d) If the $\sigma$-algebra $\mathcal{B}$ on $\Sigma$ has a countable generator, then the disintegration is unique in the following sense. If $\left(\left\{\mu_{\gamma}^{\prime}\right\}_{\gamma \in \Gamma}, \mu_{x}\right)$ is another 
disintegration of the measure $\mu$ relative to $\Gamma$, then $\mu_{\gamma}=\mu_{\gamma}^{\prime}$, for $\mu_{x}$-almost every $\gamma \in \Gamma$.

3.1.1. The $\mathcal{L}^{\infty}$ and $S^{\infty}$ spaces. Let $\mathcal{S B}(\Sigma)$ be the space of Borel signed measures on $\Sigma:=M \times K$. Given $\mu \in \mathcal{S B}(\Sigma)$ denote by $\mu^{+}$and $\mu^{-}$the positive and the negative parts of its Jordan decomposition, $\mu=\mu^{+}-\mu^{-}$(see remark 3.3). Let $\pi_{x}: \Sigma \longrightarrow M$ be the projection defined by $\pi_{x}(x, y)=x$, denote by $\pi_{x *}: \mathcal{S B}(\Sigma) \rightarrow \mathcal{S B}(M)$ the pushforward map associated to $\pi_{x}$. Denote by $\mathcal{A B}$ the set of signed measures $\mu \in \mathcal{S B}(\Sigma)$ such that its associated positive and negative marginal measures, $\pi_{x *} \mu^{+}$and $\pi_{x *} \mu^{-}$, are absolutely continuous with respect to $m_{1}$, i.e.,

$$
\mathcal{A B}=\left\{\mu \in \mathcal{S B}(\Sigma): \pi_{x *} \mu^{+}<<m_{1} \text { and } \pi_{x *} \mu^{-}<<m_{1}\right\} .
$$

Given a probability measure $\mu \in \mathcal{A B}$ on $\Sigma$, Theorem 3.1 describes a disintegration $\left(\left\{\mu_{\gamma}\right\}_{\gamma}, \mu_{x}\right)$ along $\mathcal{F}^{s}$ by a family $\left\{\mu_{\gamma}\right\}_{\gamma}$ of probability measures on the stable leaves 11 and, since $\mu \in \mathcal{A B}, \mu_{x}$ can be identified with a non negative marginal density $\phi_{x}: M \longrightarrow \mathbb{R}$, defined almost everywhere, with $\left|\phi_{x}\right|_{1}=1$. For a general (non normalized) positive measure $\mu \in \mathcal{A B}$ we can define its disintegration in the same way. In this case, $\mu_{\gamma}$ are still probability measures, $\phi_{x}$ is still defined and $\left|\phi_{x}\right|_{1}=\mu(\Sigma)$.

Definition 3.2. Let $\pi_{y}: \Sigma \longrightarrow K$ be the projection defined by $\pi_{y}(x, y)=y$. Let $\gamma \in \mathcal{F}^{s}$, consider $\pi_{\gamma, y}: \gamma \longrightarrow K$, the restriction of the map $\pi_{y}: \Sigma \longrightarrow K$ to the vertical leaf $\gamma$, and the associated pushforward map $\pi_{\gamma, y *}$. Given a positive measure $\mu \in \mathcal{A B}$ and its disintegration along the stable leaves $\mathcal{F}^{s}$, $\left(\left\{\mu_{\gamma}\right\}_{\gamma}, \mu_{x}=\phi_{x} m_{1}\right)$, we define the restriction of $\mu$ on $\gamma$ and denote it by $\left.\mu\right|_{\gamma}$ as the positive measure on $K$ (not on the leaf $\gamma$ ) defined, for all mensurable set $A \subset K$, as

$$
\left.\mu\right|_{\gamma}(A)=\pi_{\gamma, y *}\left(\phi_{x}(\gamma) \mu_{\gamma}\right)(A) .
$$

For a given signed measure $\mu \in \mathcal{A B}$ and its Jordan decomposition $\mu=$ $\mu^{+}-\mu^{-}$, define the restriction of $\mu$ on $\gamma$ by

$$
\left.\mu\right|_{\gamma}=\left.\mu^{+}\right|_{\gamma}-\left.\mu^{-}\right|_{\gamma} \text {. }
$$

Remark 3.3. As proved in Appendix 2 of [21], the restriction $\left.\mu\right|_{\gamma}$ does not depend on the decomposition. Precisely, if $\mu=\mu_{1}-\mu_{2}$, where $\mu_{1}$ and $\mu_{2}$ are any positive measures, then $\left.\mu\right|_{\gamma}=\left.\mu_{1}\right|_{\gamma}-\left.\mu_{2}\right|_{\gamma} m_{1}$-a.e. $\gamma \in M$.

Let $(X, d)$ be a compact metric space, $g: X \longrightarrow \mathbb{R}$ be a $\zeta$-Hölder function and let $H_{\zeta}(g)$ be its best $\zeta$-Hölder's constant, i.e.,

$$
H_{\zeta}(g)=\sup _{x, y \in X, x \neq y}\left\{\frac{|g(x)-g(y)|}{d(x, y)^{\zeta}}\right\} \text {. }
$$

\footnotetext{
${ }^{1}$ In the following to simplify notations, when no confusion is possible we will indicate the generic leaf or its coordinate with $\gamma$.
} 
In what follows, we generalize the definition of the Wasserstein-Kantorovichlike metric given in 21] and [23]. This generalization allows us to obtain exponential decay of correlations on the set of $\zeta$-Hölder, instead of Lipschitz, functions.

Definition 3.4. Given two signed measures $\mu$ and $\nu$ on $X$, we define a Wasserstein-Kantorovich-like distance between $\mu$ and $\nu$ by

$$
W_{1}^{\zeta}(\mu, \nu)=\sup _{H_{\zeta}(g) \leq 1,|g|_{\infty} \leq 1}\left|\int g d \mu-\int g d \nu\right| .
$$

Since the constant $\zeta$ is fixed, from now on we denote

$$
\|\mu\|_{W}:=W_{1}^{\zeta}(0, \mu) \text {. }
$$

As a matter of fact, $\|\cdot\|_{W}$ defines a norm on the vector space of signed measures defined on a compact metric space. It is worth to remark that this norm is equivalent to the dual of the $\zeta$-Hölder norm.

Other applications of this metric to obtain limit theorems can be seen in [28] and [30]. For instance, in [28] the author apply this metric to a more general case of shrinking fibers systems.

Definition 3.5. Let $\mathcal{L}^{\infty} \subseteq \mathcal{A B}(\Sigma)$ be defined as

$$
\mathcal{L}^{\infty}=\left\{\mu \in \mathcal{A B}: \operatorname{ess} \sup \left(W_{1}^{\zeta}\left(\left.\mu^{+}\right|_{\gamma},\left.\mu^{-}\right|_{\gamma}\right)\right)<\infty\right\},
$$

where the essential supremum is taken over $M$ with respect to $m_{1}$. Define the function $\|\cdot\|_{\infty}: \mathcal{L}^{\infty} \longrightarrow \mathbb{R}$ by

$$
\|\mu\|_{\infty}=\operatorname{ess} \sup \left(W_{1}^{\zeta}\left(\left.\mu^{+}\right|_{\gamma},\left.\mu^{-}\right|_{\gamma}\right)\right) \text {. }
$$

Finally, consider the following set of signed measures on $\Sigma$

$$
S^{\infty}=\left\{\mu \in \mathcal{L}^{\infty} ; \phi_{x} \in H_{\zeta}\right\},
$$

and the function, $\|\cdot\|_{S^{\infty}}: S^{\infty} \longrightarrow \mathbb{R}$, defined by

$$
\|\mu\|_{S^{\infty}}=\left|\phi_{x}\right|_{\zeta}+\|\mu\|_{\infty} \text {. }
$$

The proof of the next proposition is straightforward and can be found in 34].

Proposition 3.6. $\left(\mathcal{L}^{\infty},\|\cdot\|_{\infty}\right)$ and $\left(S^{\infty},\|\cdot\|_{S^{\infty}}\right)$ are normed vector spaces.

\section{The transfer operator ASSOCiated to $F$}

In this section, we consider the transfer operator associated to skew product maps as defined in Section 2, acting on our disintegrated measures spaces defined in Section 3. For such transfer operators and measures we prove a kind of Perron-Frobenius formula, which is somewhat similar to the one used for one-dimensional maps.

Consider the pushforward map $\mathrm{F}_{*}$ associated with $F$, defined by

$$
\left[\mathrm{F}_{*} \mu\right](E)=\mu\left(F^{-1}(E)\right),
$$


for each signed measure $\mu \in \mathcal{S B}(\Sigma)$ and for each measurable set $E \subset \Sigma$, where $\Sigma:=M \times K$. When $\mathrm{F}_{*}$ acts on the vector space $\mathcal{S B}(\Sigma)$ or on suitable vector subspaces of more regular measures, $\mathrm{F}_{*}$ is a linear map. For this reason, we also call it "transfer operator associated to $F$ ".

Lemma 4.1. For every probability $\mu \in \mathcal{A B}$ disintegrated by $\left(\left\{\mu_{\gamma}\right\}_{\gamma}, \phi_{x}\right)$, the disintegration $\left(\left\{\left(\mathrm{F}_{*} \mu\right)_{\gamma}\right\}_{\gamma},\left(\mathrm{F}_{*} \mu\right)_{x}\right)$ of the pushforward $\mathrm{F}_{*} \mu$ satisfies the following relations

$$
\left(\mathrm{F}_{*} \mu\right)_{x}=\mathrm{P}_{f}\left(\phi_{x}\right) m_{1}
$$

and

$$
\left(\mathrm{F}_{*} \mu\right)_{\gamma}=\nu_{\gamma}:=\frac{1}{\mathrm{P}_{f}\left(\phi_{x}\right)(\gamma)} \sum_{i=1}^{\operatorname{deg}(f)} \frac{\phi_{x}}{\left|\operatorname{det} D f_{i}\right|} \circ f_{i}^{-1}(\gamma) \cdot \chi_{f_{i}\left(P_{i}\right)}(\gamma) \cdot \mathrm{F}_{*} \mu_{f_{i}^{-1}(\gamma)}
$$

when $\mathrm{P}_{f}\left(\phi_{x}\right)(\gamma) \neq 0$. Otherwise, if $\mathrm{P}_{f}\left(\phi_{x}\right)(\gamma)=0$, then $\nu_{\gamma}$ is the Lebesgue measure on $\gamma$ (the expression $\frac{\phi_{x}}{\left|\operatorname{det} D f_{i}\right|} \circ f_{i}^{-1}(\gamma) \cdot \frac{\chi_{f_{i}\left(P_{i}\right)}(\gamma)}{\mathrm{P}_{f}\left(\phi_{x}\right)(\gamma)} \cdot \mathrm{F}_{*} \mu_{f_{i}^{-1}(\gamma)}$ is understood to be zero outside $f_{i}\left(P_{i}\right)$ for all $\left.i=1, \cdots, \operatorname{deg}(f)\right)$. Here and above, $\chi_{A}$ is the characteristic function of the set $A$.

Proof. By the uniqueness of the disintegration (see Theorem 3.1 ) it is enough to prove the following equation

$$
\mathrm{F}_{*} \mu(E)=\int_{M} \nu_{\gamma}(E \cap \gamma) \mathrm{P}_{f}\left(\phi_{x}\right)(\gamma) d m_{1}(\gamma)
$$

for a measurable set $E \subset \Sigma$. For this purpose, let us define the set $B_{1}=$ $\left\{\gamma ; \mathrm{P}_{f}\left(\phi_{x}\right)(\gamma)=0\right\}$. It is not hard to see that $\phi_{x} m_{1}\left(f^{-1}\left(B_{1}\right)\right)=0$. 
Using the change of variables $\gamma=f_{i}(\beta)$ and the definition of $\nu_{\gamma}$ (see (17)), we have

$$
\begin{aligned}
& \int_{M} \nu_{\gamma}(E \cap \gamma) \mathrm{P}_{f}\left(\phi_{x}\right)(\gamma) d m_{1}(\gamma) \\
& =\int_{B_{1}^{c}} \sum_{i=1}^{\operatorname{deg}(f)} \frac{\phi_{x}}{\left|\operatorname{det} D f_{i}\right|} \circ f_{i}^{-1}(\gamma) \cdot \chi_{f_{i}\left(P_{i}\right)}(\gamma) \cdot \mathrm{F}_{*} \mu_{f_{i}^{-1}(\gamma)} d m_{1}(\gamma) \\
& =\sum_{i=1}^{\operatorname{deg}(f)} \int_{f_{i}\left(P_{i}\right) \cap B_{1}^{c}} \frac{\phi_{x}}{\left|\operatorname{det} D f_{i}\right|} \circ f_{i}^{-1}(\gamma) \mathrm{F}_{*} \mu_{f_{i}^{-1}(\gamma)}(E) d m_{1}(\gamma) \\
& =\sum_{i=1}^{\operatorname{deg}(f)} \int_{P_{i} \cap f_{i}^{-1}\left(B_{1}^{c}\right)} \phi_{x}(\beta) \mu_{\beta}\left(F^{-1}(E)\right) d m_{1}(\beta) \\
& =\int_{f^{-1}\left(B_{1}^{c}\right)} \phi_{x}(\beta) \mu_{\beta}\left(F^{-1}(E)\right) d m_{1}(\beta) \\
& =\int_{M} \mu_{\beta}\left(F^{-1}(E)\right) d \phi_{x} m_{1}(\beta) \\
& =\mu\left(F^{-1}(E)\right) \\
& =\mathrm{F}_{*} \mu(E) \text {. }
\end{aligned}
$$

As said in Remark 3.3, the restriction $\left.\mu\right|_{\gamma}$ does not depend on the decomposition. Thus, for each $\mu \in \mathcal{L}^{\infty}$, since $\mathrm{F}_{*} \mu$ can be decomposed as $\mathrm{F}_{*} \mu=\mathrm{F}_{*}\left(\mu^{+}\right)-\mathrm{F}_{*}\left(\mu^{-}\right)$, we can apply the above Lemma to $\mathrm{F}_{*}\left(\mu^{+}\right)$and $\mathrm{F}_{*}\left(\mu^{-}\right)$to get the following.

Proposition 4.2. Let $\gamma \in \mathcal{F}^{s}$ be a stable leaf. Let us define the map $F_{\gamma}$ : $K \longrightarrow K$ by

$$
F_{\gamma}=\left.\pi_{y} \circ F\right|_{\gamma} \circ \pi_{\gamma, y}^{-1}
$$

Then, for each $\mu \in \mathcal{L}^{\infty}$ and for almost all $\gamma \in M$ (interpreted as the quotient space of leaves) it holds

$$
\left.\left(\mathrm{F}_{*} \mu\right)\right|_{\gamma}=\left.\sum_{i=1}^{\operatorname{deg}(f)} \mathrm{F}_{\gamma_{i} *} \mu\right|_{\gamma_{i}} \rho_{i}\left(\gamma_{i}\right) \chi_{f_{i}\left(P_{i}\right)}(\gamma) m_{1}-\text { a.e. } \quad \gamma \in M
$$

where $\mathrm{F}_{\gamma_{i} *}$ is the pushforward map associated to $\mathrm{F}_{\gamma_{i}}, \gamma_{i}=f_{i}^{-1}(\gamma)$ when $\gamma \in f_{i}\left(P_{i}\right)$ and $\rho_{i}(\gamma)=\frac{1}{\left|\operatorname{det}\left(f_{i}^{\prime}(\gamma)\right)\right|}$, where $f_{i}=\left.f\right|_{P_{i}}$.

Sometimes (see also Remark 2.2) it will be convenient to use the following expression for $\left.\left(\mathrm{F}_{*} \mu\right)\right|_{\gamma}$ :

Corollary 4.3. For each $\mu \in \mathcal{L}^{\infty}$ it holds

$$
\left.\left(\mathrm{F}_{*} \mu\right)\right|_{\gamma}=\left.\sum_{i=1}^{\operatorname{deg}(f)} \mathrm{F}_{\gamma_{i} *} \mu\right|_{\gamma_{i}} \rho_{i}\left(\gamma_{i}\right) \quad m_{1} \text {-a.e. } \gamma \in M,
$$


where $\gamma_{i}$ is the $i$-th pre image of $\gamma, i=1, \cdots, \operatorname{deg}(f)$.

\section{BASIC PROPERTIES OF THE NORMS AND CONVERGENCE TO EQUILIBRIUM}

In this section, we show important properties of the norms and their behaviour with respect to the transfer operator. In particular, we prove that the $\mathcal{L}^{\infty}$ norm is weakly contracted. We also prove other properties, like a Lasota-Yorke inequality for the strong norm and exponential convergence to equilibrium. All these properties will be used in next section to prove the spectral gap for the transfer operator associated to the system $F: \Sigma \rightarrow \Sigma$.

Proposition 5.1 (The weak norm is weakly contracted by $\mathrm{F}_{*}$ ). If $\mu \in \mathcal{L}^{\infty}$ then

$$
\left\|\mathrm{F}_{*} \mu\right\|_{\infty} \leq\|\mu\|_{\infty}
$$

In the proof of the proposition we will use the following lemma about the behaviour of the $\|\cdot\|_{W}$ norm (see equation (14)) which says that a contraction cannot increase the $\|\cdot\|_{W}$ norm.

Lemma 5.2. For every $\mu \in \mathcal{A B}$ and a stable leaf $\gamma \in \mathcal{F}^{s}$, it holds

$$
\left\|\left.\mathrm{F}_{\gamma *} \mu\right|_{\gamma}\right\|_{W} \leq\left\|\left.\mu\right|_{\gamma}\right\|_{W},
$$

where $F_{\gamma}: K \longrightarrow K$ is defined in Proposition 4.2 and $\mathrm{F}_{\gamma *}$ is the associated pushforward map. Moreover, if $\mu$ is a probability measure on $K$, it holds

$$
\left\|\mathrm{F}_{\gamma *}{ }^{n} \mu\right\|_{W}=\|\mu\|_{W}=1, \quad \forall n \geq 1 .
$$

Proof. (of Lemma 5.2) Indeed, since $F_{\gamma}$ is an $\alpha$-contraction, if $|g|_{\infty} \leq 1$ and $H_{\zeta}(g) \leq 1$, the same holds for $g \circ F_{\gamma}$. Since

$$
\left.\left|\int g d \mathrm{~F}_{\gamma *} \mu\right|_{\gamma}|=| \int g\left(F_{\gamma}\right) d \mu\right|_{\gamma} \mid
$$

taking the supremum over $g$ such that $|g|_{\infty} \leq 1$ and $H_{\zeta}(g) \leq 1$ we finish the proof of the inequality 21 .

In order to prove equation (22), consider a probability measure $\mu$ on $K$ and a $\zeta$-Hölder function $g: K \longrightarrow \mathbb{R}$, such that $\|g\|_{\infty} \leq 1$. We get immediately $\left|\int g d \mu\right| \leq\|g\|_{\infty} \leq 1$, which yields $\|\mu\|_{W} \leq 1$. Considering $g \equiv 1$, we get $\|\mu\|_{W}=1$.

Proof. (of Proposition 5.1) 
Lemma 5.2 and Corollary 4.3 yield

$$
\begin{aligned}
\left\|\left.\left(\mathrm{F}_{*} \mu\right)\right|_{\gamma}\right\|_{W} & \leq \sum_{i=1}^{\operatorname{deg}(f)}\left\|\left.\mathrm{F}_{\gamma_{i} *} \mu\right|_{\gamma_{i}} \rho_{i}\left(\gamma_{i}\right)\right\|_{W} \\
& \leq \sum_{i=1}^{\operatorname{deg}(f)} \rho_{i}\left(\gamma_{i}\right)\left\|\left.\mu\right|_{\gamma_{i}}\right\|_{W} \\
& \leq\|\mu\|_{\infty} \sum_{i=1}^{\operatorname{deg}(f)} \rho_{i}\left(\gamma_{i}\right) \\
& =\|\mu\|_{\infty} .
\end{aligned}
$$

We finish the proof by taking the essential suppremum over $\gamma$.

The following proposition shows a regularizing action of the transfer operator with respect to the strong norm. Such inequalities are usually called Lasota-Yorke or Doeblin-Fortet inequalities.

Proposition 5.3 (Lasota-Yorke inequality for $S^{\infty}$ ). There exist $A, B_{2}>0$ and $\lambda<1$ ( $\lambda=\beta_{2}$ of Corollary 2.7) such that, for all $\mu \in S^{1}$, it holds

$$
\left\|\mathrm{F}_{*}^{n} \mu\right\|_{S^{\infty}} \leq A \lambda^{n}\|\mu\|_{S^{\infty}}+B_{2}\|\mu\|_{\infty}, \quad \forall n \geq 1 .
$$

Proof. Firstly we recall that $\phi_{x}$ is the marginal density of the disintegration of $\mu$. Precisely, $\phi_{x}=\phi_{x}^{+}-\phi_{x}^{-}$, where $\phi_{x}^{+}=\frac{d \pi_{x}^{*} \mu^{+}}{d m_{1}}$ and $\phi_{x}^{-}=\frac{d \pi_{x}^{*} \mu^{-}}{d m_{1}}$. By the definition of the Wasserstein norm, it follows that for every $\gamma$ it holds $\left.|| \mu\right|_{\gamma} \|_{W} \geq\left|\int 1 d\left(\left.\mu\right|_{\gamma}\right)\right|=\left|\phi_{x}(\gamma)\right|$. Thus, $\left|\phi_{x}\right|_{\infty} \leq\|\mu\|_{\infty}$. By this last remark, equation (12) and Proposition 5.1 we have

$$
\begin{aligned}
\left\|\mathrm{F}_{*}^{n} \mu\right\|_{S^{\infty}} & =\left|\mathrm{P}_{f}^{n} \phi_{x}\right|_{\zeta}+\left\|\mathrm{F}_{*}^{n} \mu\right\|_{\infty} \\
& \leq B_{3} \beta_{2}^{n}\left|\phi_{x}\right|_{\zeta}+C_{2}\left|\phi_{x}\right|_{\infty}+\|\mu\|_{\infty} \\
& \leq B_{3} \beta_{2}^{n}|| \mu\left\|_{S^{\infty}}+\left(C_{2}+1\right)\right\| \mu \|_{\infty} .
\end{aligned}
$$

We finish the proof by setting $\lambda=\beta_{2}, A=B_{3}$ and $B_{2}=C_{2}+1$.

5.1. Convergence to equilibrium. Let $X$ be a compact metric space. Consider the space $\mathcal{S B}(X)$ of signed Borel measures on $X$. In the following, we consider two further normed vectors spaces of signed Borel measures on $X$. The spaces $\left(B_{s},\|\|_{s}\right) \subseteq\left(B_{w},\|\|_{w}\right) \subseteq \mathcal{S B}(X)$ with norms satisfying

$$
\|\|_{w} \leq\|\|_{s} .
$$

We say that a Markov operator

$$
\mathrm{L}: B_{w} \longrightarrow B_{w}
$$

has convergence to equilibrium with speed at least $\Phi$ and with respect to the norms $\|\cdot\|_{s}$ and $\|\cdot\|_{w}$, if for each $\mu \in \mathcal{V}_{s}$, where

$$
\mathcal{V}_{s}=\left\{\mu \in B_{s}, \mu(X)=0\right\}
$$


is the space of zero-average measures, it holds

$$
\left\|\mathrm{L}^{n}(\mu)\right\|_{w} \leq \Phi(n)|| \mu \|_{s},
$$

where $\Phi(n) \longrightarrow 0$ as $n \longrightarrow \infty$.

In this section, we prove that $F_{*}$ has exponential convergence to equilibrium. This is weaker than spectral gap. However, the spectral gap follows from the above Lasota-Yorke inequality and the convergence to equilibrium. Before the main statements we need some preliminary lemmata. The following is somewhat similar to Lemma 5.2 considering the behaviour of the $\|\cdot\|_{W}$ norm after a contraction. It gives a finer estimate for zero average measures and it is useful to estimate the behaviour of our $W$ norms under contractions.

Lemma 5.4. For all signed measures $\mu$ on $K$ and for all $\gamma \in \mathcal{F}^{s}$, it holds

$$
\left\|\mathrm{F}_{\gamma *} \mu\right\|_{W} \leq \alpha^{\zeta}\|\mu\|_{W}+\mu(K)
$$

( $\alpha$ is the rate of contraction of $G$, see (6) ). In particular, if $\mu(K)=0$ then

$$
\left\|\mathrm{F}_{\gamma *} \mu\right\|_{W} \leq \alpha^{\zeta}\|\mu\|_{W}
$$

Proof. If $H_{\zeta}(g) \leq 1$ and $\|g\|_{\infty} \leq 1$, then $g \circ F_{\gamma}$ is $\alpha^{\zeta}$-Hölder. Moreover, since $\|g\|_{\infty} \leq 1$, then $\left\|g \circ F_{\gamma}-\theta\right\|_{\infty} \leq \alpha^{\zeta}$, for some $\theta$ such that $|\theta| \leq 1$. Indeed, let $z \in K$ be such that $\left|g \circ F_{\gamma}(z)\right| \leq 1$, set $\theta=g \circ F_{\gamma}(z)$ and let $d_{2}$ be the Riemannian metric of $K$. Since $\operatorname{diam}(K)=1$, we have

$$
\left|g \circ F_{\gamma}(y)-\theta\right| \leq \alpha^{\zeta} d_{2}(y, z) \leq \alpha^{\zeta}
$$

and consequently $\left\|g \circ F_{\gamma}-\theta\right\|_{\infty} \leq \alpha^{\zeta}$.

This implies

$$
\begin{aligned}
\left|\int_{K} g d \mathrm{~F}_{\gamma *} \mu\right| & =\left|\int_{K} g \circ F_{\gamma} d \mu\right| \\
& \leq\left|\int_{K} g \circ F_{\gamma}-\theta d \mu\right|+\left|\int_{K} \theta d \mu\right| \\
& =\alpha^{\zeta}\left|\int_{K} \frac{g \circ F_{\gamma}-\theta}{\alpha^{\zeta}} d \mu\right|+|\theta||\mu(K)| .
\end{aligned}
$$

Taking the supremum over $g$ such that $|g|_{\infty} \leq 1$ and $H_{\zeta}(g) \leq 1$, we have $\left\|\mathrm{F}_{\gamma^{*}} \mu\right\|_{W} \leq \alpha^{\zeta}\|\mu\|_{W}+\mu(K)$. In particular, if $\mu(K)=0$, we get the second part.

Now we are ready to show a key estimate regarding the behaviour of our weak $\|\cdot\|_{\infty}$ norm for the systems defined at beginning of Section 2.1.

Proposition 5.5. For every signed measure $\mu \in \mathcal{L}^{\infty}$, it holds

$$
\left\|\mathrm{F}_{*} \mu\right\|_{\infty} \leq \alpha^{\zeta}\|\mu\|_{\infty}+\left|\phi_{x}\right|_{\infty} .
$$


Proof. Let $f_{i}$ be the branches of $f$, for all $i=1 \cdots \operatorname{deg}(f)$. Applying Lemma 5.4 on the third line below, we have

$$
\begin{aligned}
\left\|\left.\left(\mathrm{F}_{*} \mu\right)\right|_{\gamma}\right\|_{W} & =\left\|\left.\sum_{i=1}^{\operatorname{deg}(f)} \mathrm{F}_{\gamma_{i}} * \mu\right|_{\gamma_{i}} \rho\left(\gamma_{i}\right)\right\|_{W} \\
& \leq \sum_{i=1}^{\operatorname{deg}(f)}\left\|\left.\mathrm{F}_{\gamma_{i}} * \mu\right|_{\gamma_{i}} \rho\left(\gamma_{i}\right)\right\|_{W} \\
& \leq \sum_{i=1}^{\operatorname{deg}(f)}\left(\alpha^{\zeta}\left\|\left.\mu\right|_{\gamma_{i}}\right\|_{W}+\left|\phi_{x}\left(\gamma_{i}\right)\right|\right) \rho\left(\gamma_{i}\right) \\
& \leq\left(\alpha^{\zeta}\|\mu\|_{\infty}+\left|\phi_{x}\right|_{\infty}\right) \sum_{i=1}^{\operatorname{deg}(f)} \rho\left(\gamma_{1}\right) \\
& =\alpha^{\zeta}\|\mu\|_{\infty}+\left|\phi_{x}\right|_{\infty} .
\end{aligned}
$$

Hence, by taking the supremum on $\gamma$, we finish the proof of the statement.

Iterating (25) we get the following corollary.

Corollary 5.6. For every signed measure $\mu \in \mathcal{L}^{\infty}$ it holds

$$
\left\|\mathrm{F}_{*}^{n} \mu\right\|_{\infty} \leq\left(\alpha^{\zeta}\right)^{n}\|\mu\|_{\infty}+\bar{\alpha}\left|\phi_{x}\right|_{\infty}
$$

where $\bar{\alpha}=\frac{1}{1-\alpha^{\zeta}}$.

Let us consider the set of zero average measures in $S^{\infty}$ defined by

$$
\mathcal{V}_{s}=\left\{\mu \in S^{\infty}: \mu(\Sigma)=0\right\} .
$$

Note that, for all $\mu \in \mathcal{V}_{s}$ we have $\pi_{x *} \mu(M)=0$. Moreover, since $\pi_{x *} \mu=$ $\phi_{x} m_{1}\left(\phi_{x}=\phi_{x}^{+}-\phi_{x}^{-}\right)$, we have $\int_{M} \phi_{x} d m_{1}=0$. This allows us to apply Theorem 2.1 in the proof of the next proposition.

Proposition 5.7 (Exponential convergence to equilibrium). There exist $D_{2} \in \mathbb{R}$ and $0<\beta_{1}<1$ such that for every signed measure $\mu \in \mathcal{V}_{s}$, it holds

$$
\left\|\mathrm{F}_{*}^{n} \mu\right\|_{\infty} \leq D_{2} \beta_{1}^{n}\|\mu\|_{S^{\infty}},
$$

for all $n \geq 1$, where $\beta_{1}=\max \left\{\sqrt{r}, \sqrt{\alpha^{\zeta}}\right\}$ and $D_{2}=\left({\sqrt{\alpha^{\zeta}}}^{-1}+\bar{\alpha} D \sqrt{r}^{-1}\right)$.

Proof. In this proof, to simplify the notation, we denote the constant $\alpha^{\zeta}$ just by $\alpha$.

Given $\mu \in \mathcal{V}_{s}$ and denoting $\phi_{x}=\phi_{x}^{+}-\phi_{x}^{-}$, it holds that $\int \phi_{x} d m_{1}=0$. Moreover, Theorem 2.1 yields $\left|\mathrm{P}_{f}^{n}\left(\phi_{x}\right)\right|_{\zeta} \leq D r^{n}\left|\phi_{x}\right|_{\zeta}$ for all $n \geq 1$, then (since $\left.\left|\phi_{x}\right|_{\infty} \leq\|\mu\|_{\infty}\right)\left|\mathrm{P}_{f}^{n}\left(\phi_{x}\right)\right|_{\zeta} \leq D r^{n}\|\mu\|_{S^{\infty}}$, for all $n \geq 1$.

Let $l$ and $0 \leq d \leq 1$ be the coefficients of the division of $n$ by 2 , i.e., $n=2 l+d$. Thus, $l=\frac{n-d}{2}$ (by Proposition 5.1, we have $\left\|\mathrm{F}_{*}^{n} \mu\right\|_{\infty} \leq\|\mu\|_{\infty}$, 
for all $n$, and $\|\mu\|_{\infty} \leq\|\mu\|_{S^{\infty}}$ ) and by Corollary [5.6, it holds (below, set $\left.\beta_{1}=\max \left\{\sqrt{r}, \sqrt{\alpha^{\zeta}}\right\}\right)$

$$
\begin{aligned}
\left\|\mathrm{F}_{*}^{n} \mu\right\|_{\infty} & =\left\|\mathrm{F}_{*}^{2 l+d} \mu\right\|_{\infty} \\
& \leq\left(\alpha^{\zeta}\right)^{l}\left\|\mathrm{~F}_{*}^{l+d} \mu\right\|_{\infty}+\bar{\alpha}\left|\frac{d\left(\pi_{x *}\left(\mathrm{~F}_{*}^{l+d} \mu\right)\right)}{d m_{1}}\right|_{\infty} \\
& \leq\left(\alpha^{\zeta}\right)^{l}\|\mu\|_{\infty}+\bar{\alpha}\left|\mathrm{P}_{f}^{l}\left(\phi_{x}\right)\right|_{\infty} \\
& \leq\left({\sqrt{\alpha^{\zeta}}}^{-1}+\bar{\alpha} D \sqrt{r}^{-1}\right) \beta_{1}^{n}\|\mu\|_{S^{\infty}} \\
& \leq D_{2} \beta_{1}^{n} \mid\|\mu\|_{S^{\infty}}
\end{aligned}
$$

where $D_{2}=\left({\sqrt{\alpha^{\zeta}}}^{-1}+\bar{\alpha} D \sqrt{r}^{-1}\right)$.

The next lemma 5.8 ensures the existence and uniqueness of an $F$-invariant measure which projects on $m_{1}$. Since its proof is done by standard arguments (see [3], for instance) we skip it.

Lemma 5.8. There exists an unique measure $\mu_{0}$ on $M \times K$ such that for every continuous function $\psi \in C^{0}(M \times K)$ it holds

$$
\lim \int \inf _{\gamma \times K} \psi \circ F^{n} d m_{1}(\gamma)=\lim \int \sup _{\gamma \times K} \psi \circ F^{n} d m_{1}(\gamma)=\int \psi d \mu_{0} .
$$

Moreover, the measure $\mu_{0}$ is F-invariant and $\pi_{x *} \mu_{0}=m_{1}$.

Now we present the proof of Theorem $\mathrm{A}$ which says that the system has a unique invariant measure $\mu_{0} \in S^{\infty}$.

Proof. of Theorem A

Let $\mu_{0}$ be the $F$-invariant measure such that $\pi_{x *} \mu_{0}=m_{1}$ (which do exist by Lemma [5.8), where 1 is the unique $f$-invariant density in $H_{\zeta}$. Suppose that $g: K \longrightarrow \mathbb{R}$ is a $\zeta$-Hölder function such that $|g|_{\infty} \leq 1$ and $H_{\zeta}(g) \leq 1$. Then, it holds $\left|\int g d\left(\left.\mu_{0}\right|_{\gamma}\right)\right| \leq|g|_{\infty} \leq 1$. Hence, $\mu_{0} \in \mathcal{L}^{\infty}$. Since, $\frac{\pi_{x *} \mu_{0}}{d m_{1}} \equiv 1$, we have $\mu_{0} \in S^{\infty}$.

The uniqueness follows directly from Proposition 5.7, since the difference between two probabilities $\left(\mu_{1}-\mu_{0}\right)$ is a zero average signed measure.

\section{Spectral GaP}

In this section, we prove a spectral gap result for the transfer operator applied to our strong space, Theorem B. For this, we will directly use the properties proved in the previous section, and this will give a kind of constructive proof. We remark that we cannot apply the traditional Hennion, or Ionescu-Tulcea and Marinescu's approach to our function spaces because there is no compact immersion of the strong space into the weak one. This comes from the fact that we are considering the same "dual of 
Hölder" distance (see Definition 3.4) in the contracting direction for both spaces.

Proof. of Theorem B

First, let us show there exist $0<\xi<1$ and $K_{1}>0$ such that, for all $n \geq 1$, it holds

$$
\left\|\mathrm{F}_{*}^{n}\right\| \mathcal{\nu}_{s} \rightarrow \mathcal{V}_{s} \leq \xi^{n} K_{1}
$$

where $\mathcal{V}_{s}$ is the zero average space defined in (26). Indeed, consider $\mu \in \mathcal{V}_{s}$ (see (26)) s.t. $\|\mu\|_{S^{\infty}} \leq 1$ and for a given $n \in \mathbb{N}$ let $m$ and $0 \leq d \leq 1$ be the coefficients of the division of $n$ by 2, i.e., $n=2 m+d$. Thus $m=$ $\frac{n-d}{2}$. By the Lasota-Yorke inequality (Proposition 5.3) we have the uniform bound $\left\|\mathrm{F}_{*}^{n} \mu\right\|_{S^{\infty}} \leq B_{2}+A$ for all $n \geq 1$. Moreover, by Propositions 5.7 and 5.1 there is some $D_{2}$ such that it holds (below, let $\lambda_{0}$ be defined by $\left.\lambda_{0}=\max \left\{\beta_{1}, \lambda\right\}\right)$

$$
\begin{aligned}
\left\|\mathrm{F}_{*}^{n} \mu\right\|_{S^{\infty}} & \leq A \lambda^{m}\left\|\mathrm{~F}_{*}^{m+d} \mu\right\|_{S^{\infty}}+B_{2}\left\|\mathrm{~F}_{*}^{m+d} \mu\right\|_{\infty} \\
& \leq \lambda^{m} A\left(A+B_{2}\right)+B_{2}\left\|\mathrm{~F}_{*}^{m} \mu\right\|_{\infty} \\
& \leq \lambda^{m} A\left(A+B_{2}\right)+B_{2} D_{2} \beta_{1}^{m} \\
& \leq \lambda_{0}^{m}\left[A\left(A+B_{2}\right)+B_{2} D_{2}\right] \\
& \leq \lambda_{0}^{\frac{n-d}{2}}\left[A\left(A+B_{2}\right)+B_{2} D_{2}\right] \\
& \leq\left(\sqrt{\lambda_{0}}\right)^{n}\left(\frac{1}{\lambda_{0}}\right)^{\frac{d}{2}}\left[A\left(A+B_{2}\right)+B_{2} D_{2}\right] \\
& \leq \xi^{n} K_{1},
\end{aligned}
$$

where $\xi=\sqrt{\lambda_{0}}$ and $K_{1}=\left(\frac{1}{\lambda_{0}}\right)^{\frac{1}{2}}\left[A\left(A+B_{2}\right)+B_{2} D_{2}\right]$. Thus, we arrive at

$$
\left\|\left(\left.\mathrm{F}_{*}\right|_{\mathcal{V}_{s}}\right)^{n}\right\|_{S^{\infty} \rightarrow S^{\infty}} \leq \xi^{n} K_{1}
$$

Now, recall that $\mathrm{F}_{*}: S^{\infty} \longrightarrow S^{\infty}$ has a unique fixed point $\mu_{0} \in S^{\infty}$, which is a probability (see Theorem $\mathrm{A}$ ). Consider the operator $\mathrm{P}: S^{\infty} \longrightarrow\left[\mu_{0}\right]$ ( $\left[\mu_{0}\right]$ is the space spanned by $\left.\mu_{0}\right)$, defined by $\mathrm{P}(\mu)=\mu(\Sigma) \mu_{0}$. By definition, $\mathrm{P}$ is a projection and $\operatorname{dim} \mathrm{P}\left(S^{\infty}\right)=1$. Define the operator

$$
\mathrm{S}: S^{\infty} \longrightarrow \mathcal{V}_{s}
$$

by

$$
\mathrm{S}(\mu)=\mu-\mathrm{P}(\mu), \quad \forall \mu \in S^{\infty} .
$$

Thus, we set $\mathrm{N}=\mathrm{F}_{*} \circ \mathrm{S}$ and observe that, by definition, $\mathrm{PN}=\mathrm{NP}=0$ and $\mathrm{F}_{*}=\mathrm{P}+\mathrm{N}$. Moreover, $\mathrm{N}^{n}(\mu)=\mathrm{F}_{*}{ }^{n}(\mathrm{~S}(\mu))$ for all $n \geq 1$. Since $\mathrm{S}$ is bounded and $\mathrm{S}(\mu) \in \mathcal{V}_{s}$, we get by (28), $\left\|\mathrm{N}^{n}(\mu)\right\|_{S^{\infty}} \leq \xi^{n} K\|\mu\|_{S^{\infty}}$, for all $n \geq 1$, where $K=K_{1}\|\mathrm{~S}\|_{S^{\infty} \rightarrow S^{\infty}}$.

Remark 6.1. The constant $\xi$ for the map $F$, found in Theorem $B$, is directly related to the coefficients of the Lasota-Yorke inequality for the basis map (see Corollary 2.7) and the rate of convergence to equilibrium of $F$ (see Proposition [5.7) found before. More precisely, $\xi=\max \left\{\sqrt{\lambda}, \sqrt{\beta_{1}}\right\}$. We 
remark that, from the above proof we also have an explicit estimate for $K$ in the exponential convergence, while many classical approaches are not suitable for this.

6.1. Exponential Decay of Correlations. In this section, we present one of the standard consequences of spectral gap, Theorem C. We will show how Theorem B implies an exponential rate of convergence for the limit

$$
\lim _{n \rightarrow \infty} C_{n}(f, g)=0
$$

where

$$
C_{n}(f, g):=\left|\int\left(g \circ F^{n}\right) f d \mu_{0}-\int g d \mu_{0} \int f d \mu_{0}\right|,
$$

$g: \Sigma \longrightarrow \mathbb{R}$ is a $\zeta$-Hölder function and $f \in \Theta_{\mu_{0}}$. The sets $\Theta_{\mu_{0}}$ are defined by

$$
\Theta_{\mu_{0}}:=\left\{f: \Sigma \longrightarrow \mathbb{R} ; f \mu_{0} \in S^{\infty}\right\}
$$

where the measure $f \mu_{0}$ is defined by $f \mu_{0}(E):=\int_{E} f d \mu_{0}$ for all measurable sets $E$.

Proof. of Theorem C

Let $g: \Sigma \longrightarrow \mathbb{R}$ be a $\zeta$-Hölder function and $f \in \Theta_{\mu_{0}}$. By Theorem $\mathbb{B}$, we have

$$
\begin{aligned}
\mid \int\left(g \circ F^{n}\right) f \mu_{0}-\int g d \mu_{0} & \int f d \mu_{0}|=| \int g d \mathrm{~F}_{*}^{n}\left(f \mu_{0}\right)-\int g d \mathrm{P}\left(f \mu_{0}\right) \mid \\
& \leq\left\|\mathrm{F}_{*}^{n}\left(f \mu_{0}\right)-\mathrm{P}\left(f \mu_{0}\right)\right\|_{W} \max \left\{H_{\zeta}(g),\|g\|_{\infty}\right\} \\
& =\left\|\mathrm{N}^{n}\left(f \mu_{0}\right)\right\|_{W} \max \left\{H_{\zeta}(g),\|g\|_{\infty}\right\} \\
& \leq\left\|\mathrm{N}^{n}\left(f \mu_{0}\right)\right\|_{S^{\infty}} \max \left\{H_{\zeta}(g),\|g\|_{\infty}\right\} \\
& \leq\left\|f \mu_{0}\right\|_{S^{\infty}} K|g|_{\zeta} \xi^{n} .
\end{aligned}
$$

In Theorem E we will see, under some further assumptions on the system, that the sets $\Theta_{\mu_{0}}$ contain the set of $\zeta$-Hölder functions on $\Sigma$.

\section{HÖlDER MEASURES}

In this section we will prove Theorem D. We suppose that $G$ satisfies the additional property (G2) stated below. Moreover, besides satisfying equation (5), the constant $L$ mentioned in (f1) and (f3) is also supposed to be close enough to 1 such that $(\alpha \cdot L)^{\zeta}<1$ (or $\alpha$ is close enough to 0 ). This is not restrictive for the aimed examples and it is clearly satisfied by Example 2.1

(G2) Let $P_{1}, \cdots, P_{\operatorname{deg}(f)}$ be the partition of $M$ given in Remark 2.2, Suppose that

$$
\left|G_{i}\right|_{\zeta}:=\sup _{y} \sup _{x_{1}, x_{2} \in P_{i}} \frac{d_{2}\left(G\left(x_{1}, y\right), G\left(x_{2}, y\right)\right)}{d_{1}\left(x_{1}, x_{2}\right)^{\zeta}}<\infty .
$$


And denote by $|G|_{\zeta}$ the following constant

$$
|G|_{\zeta}:=\max _{i=1, \cdots, s}\left\{\left|G_{i}\right|_{\zeta}\right\} .
$$

Remark 7.1. The condition (G2) means that $G$ can be discontinuous on the sets $\partial P_{i} \times K$, for all $i=1, \cdots, \operatorname{deg}(f)$, where $\partial P_{i}$ denotes the boundary of $P_{i}$.

We have seen that a positive measure on $M \times K$ can be disintegrated along the stable leaves $\mathcal{F}^{s}$ in a way that we can see it as a family of positive measures on $M,\left\{\left.\mu\right|_{\gamma}\right\}_{\gamma \in \mathcal{F}^{s}}$. Since there is a one-to-one correspondence between $\mathcal{F}^{s}$ and $M$, this defines a path in the metric space of positive measures $(\mathcal{S B}(K))$ defined on $K, M \longmapsto \mathcal{S B}(K)$, where $\mathcal{S B}(K)$ is endowed with the Wasserstein-Kantorovich-like metric (see definition 3.4). It will be convenient to use a functional notation and denote such a path by $\Gamma_{\mu}: M \longrightarrow \mathcal{S B}(K)$ defined almost everywhere by $\Gamma_{\mu}(\gamma)=\left.\mu\right|_{\gamma}$, where $\left(\left\{\mu_{\gamma}\right\}_{\gamma \in M}, \phi_{x}\right)$ is some disintegration of $\mu$. However, since such a disintegration is defined $\widehat{\mu}$-a.e. $\gamma \in M$, the path $\Gamma_{\mu}$ is not unique. For this reason we define more precisely $\Gamma_{\mu}$ as the class of almost everywhere equivalent paths corresponding to $\mu$.

Definition 7.2. Consider a positive Borel measure $\mu$ on $M \times K$ and a disintegration $\omega=\left(\left\{\mu_{\gamma}\right\}_{\gamma \in M}, \phi_{x}\right)$, where $\left\{\mu_{\gamma}\right\}_{\gamma \in M}$ is a family of probabilities on $M \times K$ defined $\widehat{\mu}$-a.e. $\gamma \in M$ (where $\widehat{\mu}:=\pi_{x *} \mu=\phi_{x} m_{1}$ ) and $\phi_{x}: \Sigma_{A}^{+} \longrightarrow$ $\mathbb{R}$ is a non-negative marginal density. Denote by $\Gamma_{\mu}$ the class of equivalent paths associated to $\mu$

$$
\Gamma_{\mu}=\left\{\Gamma_{\mu}^{\omega}\right\}_{\omega},
$$

where $\omega$ ranges on all the possible disintegrations of $\mu$ and $\Gamma_{\mu}^{\omega}: M \longrightarrow$ $\mathcal{S B}(K)$ is the map associated to a given disintegration, $\omega$ :

$$
\Gamma_{\mu}^{\omega}(\gamma)=\left.\mu\right|_{\gamma}=\pi_{\gamma, y}^{*} \phi_{x}(\gamma) \mu_{\gamma} .
$$

Let us call the set on which $\Gamma_{\mu}^{\omega}$ is defined by $I_{\Gamma_{\mu}^{\omega}}(\subset M)$.

Definition 7.3. For a given $0<\zeta<1$, a disintegration $\omega$ of $\mu$ and its functional representation $\Gamma_{\mu}^{\omega}$ we define the $\zeta$-Hölder constant of $\mu$ associated to $\omega$ by

$$
|\mu|_{\zeta}^{\omega}:=\operatorname{ess} \sup _{\gamma_{1}, \gamma_{2} \in I_{\Gamma_{\mu}}^{\omega}}\left\{\frac{\left.|| \mu\right|_{\gamma_{1}}-\left.\mu\right|_{\gamma_{2}}||_{W}}{d_{1}\left(\gamma_{1}, \gamma_{2}\right)^{\zeta}}\right\} .
$$

Finally, we define the $\zeta$-Hölder constant of the positive measure $\mu$ by

$$
|\mu|_{\zeta}:=\inf _{\Gamma_{\mu}^{\omega} \in \Gamma_{\mu}}\left\{|\mu|_{\zeta}^{\omega}\right\}
$$

Remark 7.4. When no confusion is possible, to simplify the notation, we denote $\Gamma_{\mu}^{\omega}(\gamma)$ just by $\left.\mu\right|_{\gamma}$. 
Definition 7.5. From the Definition 7.3 we define the set of the $\zeta$-Hölder positive measures $\mathcal{H}_{\zeta}^{+}$as

$$
\mathcal{H}_{\zeta}^{+}=\left\{\mu \in \mathcal{A B}: \mu \geq 0,|\mu|_{\zeta}<\infty\right\} .
$$

For the next lemma, for a given path, $\Gamma_{\mu}$ which represents the measure $\mu$, we define for each $\gamma \in I_{\Gamma_{\mu}^{\omega}} \subset M$, the map

$$
\mu_{F}(\gamma):=\left.\mathrm{F}_{\gamma_{*}} \mu\right|_{\gamma}
$$

where $F_{\gamma}: K \longrightarrow K$ is defined as

$$
F_{\gamma}(y)=\pi_{y} \circ F \circ\left(\left.\pi_{y}\right|_{\gamma}\right)^{-1}(y)
$$

and $\pi_{y}: M \times K \longrightarrow K$ is the projection $\pi_{y}(x, y)=y$.

Lemma 7.6. Suppose that $F: \Sigma \longrightarrow \Sigma$ satisfies (G1) and (G2). Then, for all $\mu \in \mathcal{H}_{\zeta}^{+}$which satisfy $\phi_{x}=1 m_{1}$-a.e., it holds

$$
\left\|\left.\mathrm{F}_{x *} \mu\right|_{x}-\left.\mathrm{F}_{y *} \mu\right|_{y}\right\|_{W} \leq \alpha^{\zeta}|\mu|_{\zeta} d_{1}(x, y)^{\zeta}+|G|_{\zeta} d_{1}(x, y)^{\zeta}\|\mu\|_{\infty},
$$

for all $x, y \in P_{i}$ and all $i=1, \cdots, \operatorname{deg}(f)$.

Proof. Since $\left(\left.\mu\right|_{x}-\left.\mu\right|_{y}\right)(K)=0\left(\phi_{x}=1 m_{1}\right.$-a.e. $)$, by Lemma 5.4, it holds

$$
\begin{aligned}
\left\|\left.\mathrm{F}_{x *} \mu\right|_{x}-\left.\mathrm{F}_{y *} \mu\right|_{y}\right\|_{W} & \leq\left\|\left.\mathrm{F}_{x *} \mu\right|_{x}-\left.\mathrm{F}_{x *} \mu\right|_{y}\right\|_{W}+\left\|\left.\mathrm{F}_{x *} \mu\right|_{y}-\left.\mathrm{F}_{y *} \mu\right|_{y}\right\|_{W} \\
& \leq\left.\alpha^{\zeta}|| \mu\right|_{x}-\left.\left.\mu\right|_{y}\left\|_{W}+\right\| \mathrm{F}_{x *} \mu\right|_{y}-\left.\mathrm{F}_{y *} \mu\right|_{y} \|_{W} \\
& \leq \alpha^{\zeta}|\mu|_{\zeta} d_{1}(x, y)^{\zeta}+\left\|\left.\mathrm{F}_{x *} \mu\right|_{y}-\left.\mathrm{F}_{y *} \mu\right|_{y}\right\|_{W} .
\end{aligned}
$$

Let us estimate the second summand $\left\|\left.\mathrm{F}_{x *} \mu\right|_{y}-\left.\mathrm{F}_{y *} \mu\right|_{y}\right\|_{W}$. To do it, let $g: K \longrightarrow \mathbb{R}$ be a $\zeta$-Hölder function s.t. $H_{\zeta}(g),|g|_{\infty} \leq 1$. By equation (35), we get

$$
\begin{aligned}
\left|\int g d\left(\left.\mathrm{~F}_{x *} \mu\right|_{y}\right)-\int g d\left(\left.\mathrm{~F}_{y *} \mu\right|_{y}\right)\right| & =\mid \int g(G(x, z)) d\left(\left.\mu\right|_{y}\right)(z) \\
& -\int g(G(y, z)) d\left(\left.\mu\right|_{y}\right)(z) \mid \\
\leq & \int G(x, z)-G(y, z) \mid d\left(\left.\mu\right|_{y}\right)(z) \\
\leq & |G|_{\zeta} d_{1}(x, y)^{\zeta} \int 1 d\left(\left.\mu\right|_{y}\right)(z) \\
& \leq\left.\left.|G|_{\zeta} d_{1}(x, y)^{\zeta}|| \mu\right|_{y}\right|_{W} .
\end{aligned}
$$

Thus, taking the supremum over $g$ and the essential supremum over $y$, we get

$$
\left\|\left.\mathrm{F}_{x *} \mu\right|_{y}-\left.\mathrm{F}_{y *} \mu\right|_{y}\right\|_{W} \leq|G|_{\zeta} d_{1}(x, y)^{\zeta}\|\mu\|_{\infty} .
$$


For the next proposition and henceforth, for a given path $\Gamma_{\mu}^{\omega} \in \Gamma_{\mu}$ (associated with the disintegration $\omega=\left(\left\{\mu_{\gamma}\right\}_{\gamma}, \phi_{x}\right)$, of $\left.\mu\right)$, unless written otherwise, we consider the particular path $\Gamma_{\mathrm{F}_{*} \mu}^{\omega} \in \Gamma_{\mathrm{F}_{*} \mu}$ defined by the Corollary 4.3, by the expression

$$
\Gamma_{\mathrm{F}_{*} \mu}^{\omega}(\gamma)=\sum_{i=1}^{\operatorname{deg}(f)} \mathrm{F}_{\gamma_{i} *} \Gamma_{\mu}^{\omega}\left(\gamma_{i}\right) \rho_{i}\left(\gamma_{i}\right) \quad m_{1} \text {-a.e. } \gamma \in M .
$$

Recall that $\Gamma_{\mu}^{\omega}(\gamma)=\left.\mu\right|_{\gamma}:=\pi_{y *}\left(\phi_{x}(\gamma) \mu_{\gamma}\right)$ and in particular $\Gamma_{\mathrm{F}_{*} \mu}^{\omega}(\gamma)=$ $\left.\left(\mathrm{F}_{*} \mu\right)\right|_{\gamma}=\pi_{y *}\left(\mathrm{P}_{f} \phi_{x}(\gamma) \mu_{\gamma}\right)$, where $\phi_{x}=\frac{d \pi_{x *} \mu}{d m_{1}}$ and $\mathrm{P}_{f}$ is the PerronFrobenius operator of $f$.

Proposition 7.7. If $F: \Sigma \longrightarrow \Sigma$ satisfies (f1), (f2), (f3), (G1), (G2) and $\alpha \cdot L^{\zeta}<1$, then there exist $0<\beta<1$ and $D>0$, such that for all $\mu \in \mathcal{H}_{\zeta}^{+}$ which satisfy $\phi_{x}=1 m_{1}$-a.e. and for all $\Gamma_{\mu}^{\omega} \in \Gamma_{\mu}$, it holds

$$
\left|\Gamma_{\mathrm{F}_{*}}^{\omega} \mu\right|_{\zeta} \leq \beta\left|\Gamma_{\mu}^{\omega}\right|_{\zeta}+D|| \mu \|_{\infty},
$$

for $\beta:=(\alpha L)^{\zeta}$ and $D:=\left\{\epsilon_{\rho} L^{\zeta}+|G|_{\zeta} L^{\zeta}\right\}$.

Proof. In this proof, we denote a leaf by $x$ and $y$, instead of $\gamma$. We assume that the pre-images $x_{i}$ and $y_{i}$ belong to the same atom $P_{i}$ for all $i=1, \cdots, \operatorname{deg}(f)$, stated in (G2). This holds for $m_{1}$-a.e. $x, y \in M$. Moreover, $\sum_{i=1}^{\operatorname{deg}(f)} \rho_{i}\left(y_{i}\right)=1$, since $m_{1}$ is $f$-invariant. Note that

$$
\begin{aligned}
\left.\left(\mathrm{F}_{*} \mu\right)\right|_{x}-\left.\left(\mathrm{F}_{*} \mu\right)\right|_{y} & =\left.\sum_{i=1}^{\operatorname{deg}(f)} \mathrm{F}_{x_{i} *} \mu\right|_{x_{i}} \rho_{i}\left(x_{i}\right)-\left.\sum_{i=1}^{\operatorname{deg}(f)} \mathrm{F}_{y_{i} *} \mu\right|_{y_{i}} \rho_{i}\left(y_{i}\right) \\
& =\left.\sum_{i=1}^{\operatorname{deg}(f)} \mathrm{F}_{x_{i} *} \mu\right|_{x_{i}} \rho_{i}\left(x_{i}\right)-\left.\sum_{i=1}^{\operatorname{deg}(f)} \mathrm{F}_{x_{i} *} \mu\right|_{x_{i}} \rho_{i}\left(y_{i}\right) \\
& +\left.\sum_{i=1}^{\operatorname{deg}(f)} \mathrm{F}_{x_{i} *} \mu\right|_{x_{i}} \rho_{i}\left(y_{i}\right)-\left.\sum_{i=1}^{\operatorname{deg}(f)} \mathrm{F}_{y_{i} *} \mu\right|_{y_{i}} \rho_{i}\left(y_{i}\right) .
\end{aligned}
$$

Thus, by Lemma 5.2, we have

$$
\left\|\left.\left(\mathrm{F}_{*} \mu\right)\right|_{x}-\left.\left(\mathrm{F}_{*} \mu\right)\right|_{y}\right\|_{W} \leq \mathrm{I}_{1}+\mathrm{I}_{2}
$$

where

$$
\mathrm{I}_{1}=\left.\sum_{i=1}^{\operatorname{deg}(f)}|| \mu\right|_{x_{i}}\left|{ }_{W}\right| \rho_{i}\left(x_{i}\right)-\rho_{i}\left(y_{i}\right) \mid
$$

and

$$
\mathrm{I}_{2}=\left.\sum_{i=1}^{\operatorname{deg}(f)}\left|\rho_{i}\left(y_{i}\right)\right||| \mathrm{F}_{x_{i} *} \mu\right|_{x_{i}}-\left.\mathrm{F}_{y_{i} *} \mu\right|_{y_{i}} \|_{W} .
$$

Now, we estimate $I_{1}$ and $I_{2}$. 
By (f3), we have

$$
\begin{aligned}
\mathrm{I}_{1} & \leq \operatorname{ess}_{\sup _{x}}\left\|\left.\mu\right|_{x}\right\|_{W} \sum_{i=1}^{\operatorname{deg}(f)}\left|\rho_{i}\left(x_{i}\right)-\rho_{i}\left(y_{i}\right)\right| \\
& \leq\|\mu\|_{\infty} \sum_{i=1}^{\operatorname{deg}(f)} H_{\zeta}(\rho) d_{1}\left(x_{i}, y_{i}\right)^{\zeta} \\
& \leq\|\mu\|_{\infty} \sum_{i=1}^{\operatorname{deg}(f)} \epsilon_{\rho} \inf \rho d_{1}\left(x_{i}, y_{i}\right)^{\zeta} \\
& \leq\|\mu\|_{\infty} \epsilon_{\rho} \sum_{i=1}^{\operatorname{deg}(f)} \inf \rho d_{1}\left(x_{i}, y_{i}\right)^{\zeta} \\
& \leq\|\mu\|_{\infty} \epsilon_{\rho} \sum_{i=1}^{\operatorname{deg}(f)} \rho\left(x_{i}\right) d_{1}\left(x_{i}, y_{i}\right)^{\zeta} \\
& \leq\|\mu\|_{\infty} \epsilon_{\rho} L^{\zeta} d_{1}(x, y)^{\zeta} \sum_{i=1}^{\operatorname{deg}(f)} \rho\left(x_{i}\right) \\
& \leq\|\mu\|_{\infty} \epsilon_{\rho} L^{\zeta} d_{1}(x, y)^{\zeta} .
\end{aligned}
$$

Thus

$$
\mathrm{I}_{1} \leq\|\mu\|_{\infty} \epsilon_{\rho} L^{\zeta} d_{1}(x, y)^{\zeta} .
$$

Let us estimate $I_{2}$. Since $x_{i}, y_{i} \in P_{i}$, Lemma 7.6 yields

$$
\begin{aligned}
\mathrm{I}_{2} & =\sum_{i=1}^{\operatorname{deg}(f)}\left|\rho_{i}\left(y_{i}\right)\right|\left\|\left.\mathrm{F}_{x_{i} *} \mu\right|_{x_{i}}-\left.\mathrm{F}_{y_{i} *} \mu\right|_{y_{i}}\right\|_{W} \\
& \leq \sum_{i=1}^{\operatorname{deg}(f)} \rho_{i}\left(y_{i}\right)\left(\alpha^{\zeta}|\mu|_{\zeta} d_{1}\left(x_{i}, y_{i}\right)^{\zeta}+\left.|G|_{\zeta} d_{1}\left(x_{i}, y_{i}\right)^{\zeta}|| \mu\right|_{y_{i}} \|_{W}\right) \\
& \leq\left(\alpha^{\zeta}|\mu|_{\zeta} L^{\zeta} d_{1}(x, y)^{\zeta}+|G|_{\zeta} L^{\zeta} d_{1}(x, y)^{\zeta}\|\mu\|_{\infty}\right) \sum_{i=1}^{\operatorname{deg}(f)} \rho_{i}\left(y_{i}\right) \\
& =\alpha^{\zeta}|\mu|_{\zeta} L^{\zeta} d_{1}(x, y)^{\zeta}+|G|_{\zeta} L^{\zeta} d_{1}(x, y)^{\zeta}\|\mu\|_{\infty} .
\end{aligned}
$$

Hence

$$
\mathrm{I}_{2} \leq \alpha^{\zeta}|\mu|_{\zeta} L^{\zeta} d_{1}(x, y)^{\zeta}+|G|_{\zeta} L^{\zeta} d_{1}(x, y)^{\zeta}\|\mu\|_{\infty} .
$$

By equations (37), (40) and (41), we have

$$
\begin{gathered}
\left|\Gamma_{\mathrm{F}_{*} \mu}^{\omega}\right|_{\zeta} \leq \beta\left|\Gamma_{\mu}^{\omega}\right|_{\zeta}+D|| \mu \|_{\infty}, \\
\beta:=(\alpha L)^{\zeta} \text { and } D:=\left\{\epsilon_{\rho} L^{\zeta}+|G|_{\zeta} L^{\zeta}\right\}
\end{gathered}
$$


Iterating the inequality $\left|\Gamma_{\mathrm{F}_{*} \mu}^{\omega}\right|_{\zeta} \leq \beta\left|\Gamma_{\mu}^{\omega}\right|_{\zeta}+D\|\mu\|_{\infty}$ obtained in Proposition 17.7, a standard computation yields the next result, the proof of which is omitted.

Corollary 7.8. Suppose that $F: \Sigma \longrightarrow \Sigma$ satisfies (f1), (f2), (f3), (G1), (G2) and $(\alpha \cdot L)^{\zeta}<1$. Then, for all $\mu \in \mathcal{H}_{\zeta}^{+}$which satisfy $\phi_{x}=1 m_{1}$-a.e. and $\left\|\mathrm{F}_{*} \mu\right\|_{\infty} \leq\|\mu\|_{\infty}$, it holds

$$
\left|\Gamma_{\mathrm{F}_{*}{ }^{n} \mu}^{\omega}\right|_{\zeta} \leq \beta^{n}\left|\Gamma_{\mu}^{\omega}\right|_{\zeta}+\frac{D}{1-\beta}\|\mu\|_{\infty},
$$

for all $n \geq 1$, where $\beta$ and $D$ are from Proposition 7.7.

Remark 7.9. Taking the infimum over all paths $\Gamma_{\mu}^{\omega} \in \Gamma_{\mu}$ and all $\Gamma_{\mathrm{F}_{*}{ }^{n}{ }_{\mu}}^{\omega} \in$ $\Gamma_{\mathrm{F}_{*}{ }^{n} \mu}$ on both sides of inequality (42), we get

$$
\left|\mathrm{F}_{*}{ }^{n} \mu\right|_{\zeta} \leq \beta^{n}|\mu|_{\zeta}+\frac{D}{1-\beta}|| \mu \|_{\infty}
$$

The above Equation (43) will give a uniform bound (see the proof of Theorem 7.10) for the Hölder's constant of the measure $\mathrm{F}_{*}{ }^{n} m$, for all $n$, where $m$ is defined as the product $m=m_{1} \times \nu$, for a fixed probability measure $\nu$ on $K$. The uniform bound will be useful later on (see Theorem E).

Remark 7.10. Consider the probability measure $m$ defined in Remark 7.9. i.e., $m=m_{1} \times \nu$, where $\nu$ is a given probability measure on $K$ and $m_{1}$ is the $f$-invariant measure fixed in the subsection 2.1.1. Besides that, consider its trivial disintegration $\omega_{0}=\left(\left\{m_{\gamma}\right\}_{\gamma}, \phi_{x}\right)$, given by $m_{\gamma}=\pi_{\mathrm{y}, \gamma^{*}}^{-1} \nu$, for all $\gamma$ and $\phi_{x} \equiv 1$. According to this definition, it holds that

$$
\left.m\right|_{\gamma}=\nu, \quad \forall \gamma
$$

In other words, the path $\Gamma_{m}^{\omega_{0}}$ is constant: $\Gamma_{m}^{\omega_{0}}(\gamma)=\nu$ for all $\gamma$. Moreover, for each $n \in \mathbb{N}$, let $\omega_{n}$ be the particular disintegration of the measure $\mathrm{F}_{*}{ }^{n} m$ defined from $\omega_{0}$ as an application of Lemma 4.1, and consider the path $\Gamma_{\mathrm{F}_{*}{ }^{n} m}^{\omega_{n}}$ associated with this disintegration. By Proposition 4.2, we have

$$
\Gamma_{\mathrm{F}_{*}{ }^{n} m}^{\omega_{n}}(\gamma)=\sum_{i=1}^{q} \frac{\mathrm{F}_{\mathrm{f}_{\mathrm{i}}^{-\mathrm{n}}(\gamma)_{*}}^{\mathrm{n}} \nu}{\left.\mid \operatorname{det} D f_{i}^{n} \circ f_{i}^{-n}(\gamma)\right) \mid} \chi_{f_{i}^{n}\left(P_{i}\right)}(\gamma) m_{1}-\text { a.e } \gamma \in M,
$$

where $P_{i}, i=1, \cdots, q=q(n)$, ranges over the partition $\mathcal{P}^{(n)}$ defined in the following way: for all $n \geq 1$, let $\mathcal{P}^{(n)}$ be the partition of $I$ s.t. $\mathcal{P}^{(n)}(x)=$ $\mathcal{P}^{(n)}(y)$ if and only if $\mathcal{P}^{(1)}\left(f^{j}(x)\right)=\mathcal{P}^{(1)}\left(f^{j}(y)\right)$ for all $j=0, \cdots, n-1$, where $\mathcal{P}^{(1)}=\mathcal{P}$ (see remark 2.2). This path will be used in the proof of the next proposition.

For the next result, we recall that, by Theorem $\mathrm{A}$, $F$ has a unique invariant measure $\mu_{0} \in S^{\infty}$. We will prove that $\mu_{0}$ has a regular disintegration in a way that $\mu_{0} \in \mathcal{H}_{\zeta}$ (similar results are presented in [12, [21, [23] and [30], for others sort of systems). This will be used to prove exponential decay of correlations over the set of $\zeta$-Hölder functions. 
Proof. of Theorem D

Consider the path $\Gamma_{\mathrm{F}_{*}{ }^{\mathrm{n}}}^{\omega_{n}} m$, defined in Remark 7.10, which represents the measure $\mathrm{F}_{*}{ }^{n} m$.

According to Theorem $\mathrm{A}$, let $\mu_{0} \in S^{\infty}$ be the unique $F$-invariant probability measure in $S^{\infty}$. Consider the measure $m$, defined in Remark 7.10 and its iterates $\mathrm{F}_{*}{ }^{n}(m)$. By Theorem $\mathrm{B}$, these iterates converge to $\mu_{0}$ in $\mathcal{L}^{\infty}$. It implies that the sequence $\left\{\Gamma_{\mathrm{F}_{*}{ }^{n}(m)}^{\omega_{n}}\right\}_{n}$ converges $m_{1}$-a.e. to $\Gamma_{\mu_{0}}^{\omega} \in \Gamma_{\mu_{0}}$ (in $\mathcal{S B}(K)$ with respect to the metric defined in definition 3.4), where $\Gamma_{\mu_{0}}^{\omega}$ is a path given by the Rokhlin Disintegration Theorem and $\left\{\Gamma_{\mathrm{F}_{*}{ }^{n}(m)}^{\omega_{n}}\right\}_{n}$ is given by equation (44). It implies that $\left\{\Gamma_{\mathrm{F}_{*}{ }^{n}(m)}^{\omega_{n}}\right\}_{n}$ converges pointwise to $\Gamma_{\mu_{0}}^{\omega}$ on a full measure set $\widehat{M} \subset M$. Let us denote $\Gamma_{n}:=\left.\Gamma_{\mathrm{F}_{*}{ }^{n}(m)}^{\omega_{n}}\right|_{\widehat{M}}$ and $\Gamma:=\left.\Gamma_{\mu_{0}}^{\omega}\right|_{\widehat{M}}$. Since $\left\{\Gamma_{n}\right\}_{n}$ converges pointwise to $\Gamma$, it holds $\left|\Gamma_{n}\right|_{\zeta} \longrightarrow|\Gamma|_{\zeta}$ as $n \rightarrow \infty$. Indeed, let $x, y \in \widehat{M}$. Then,

$$
\lim _{n \longrightarrow \infty} \frac{\left\|\Gamma_{n}(x)-\Gamma_{n}(y)\right\|_{W}}{d_{1}(x, y)^{\zeta}}=\frac{\|\Gamma(x)-\Gamma(y)\|_{W}}{d_{1}(x, y)^{\zeta}} .
$$

On the other hand, by Corollary 7.8, the argument of the left hand side is bounded by $\left|\Gamma_{n}\right|_{\zeta} \leq \frac{D}{1-\beta}$ for all $n \geq 1$. Then,

$$
\frac{\|\Gamma(x)-\Gamma(y)\|_{W}}{d_{1}(x, y)^{\zeta}} \leq \frac{D}{1-\beta} .
$$

Thus, $\left|\Gamma_{\mu_{0}}^{\omega}\right|_{\zeta} \leq \frac{D}{1-\beta}$ and taking the infimum we get $\left|\mu_{0}\right|_{\zeta} \leq \frac{D}{1-\beta}$.

\section{From a Space of Measures to a Space of Functions}

In Section 6.1, we proved that systems $F: \Sigma \longrightarrow \Sigma$ satisfying (f1), (f2), (f3), (G1), (G2) and $(\alpha \cdot L)^{\zeta}<1$ have exponential decay of correlations for observables $f \in \Theta_{\mu_{0}}^{1}$ and Hölder ones. In this section, we prove that $\Theta_{\mu_{0}}^{1}$ contains the set of $\zeta$-Hölder functions, Theorem E.

Denote the space of the $\zeta$-Hölder functions, $g: \Sigma \longrightarrow \mathbb{R}$, by $\mathrm{H}_{\zeta}(\Sigma)$. As a consequence of Theorem $\mathrm{D}$, next Theorem E yields $\mathrm{H}_{\zeta}(\Sigma) \subset \Theta_{\mu_{0}}$ (defined in subsection 6.11). In order to prove it, we need the next Lemma 8.1 on disintegration of absolutely continuous measures with respect to a measure $\mu_{0} \in \mathcal{A B}$, the proof of which is postponed to the Appendix 1 (section 9).

Lemma 8.1. Let $\left(\left\{\mu_{0, \gamma}\right\}_{\gamma}, \phi_{x}\right)$ be the disintegration of $\mu_{0}$ along the partition $\mathcal{F}^{s}:=\{\{\gamma\} \times K: \gamma \in M\}$, and for a $\mu_{0}$ integrable function $h: M \times K \longrightarrow \mathbb{R}$, denote by $\nu$ the measure $\nu:=h \mu_{0}\left(h \mu_{0}(E):=\int_{E} h d \mu_{0}\right)$. If $\left(\left\{\nu_{\gamma}\right\}_{\gamma}, \widehat{\nu}\right)$ is the disintegration of $\nu$, where $\widehat{\nu}:=\pi_{x *} \nu$, then $\widehat{\nu} \ll m_{1}$ and $\nu_{\gamma} \ll \mu_{0, \gamma}$. Moreover,

$$
\frac{d \widehat{\nu}}{d m_{1}}:=\bar{h}(\gamma)=\int_{M} h(\gamma, y) d\left(\left.\mu_{0}\right|_{\gamma}\right)
$$


and for $\widehat{\nu}$-a.e. $\gamma \in M$ and all $y \in K$,

$$
\frac{d \nu_{\gamma}}{d \mu_{0, \gamma}}(y)=\left\{\begin{array}{cc}
\frac{\left.h\right|_{\gamma}(y)}{\left.\int h\right|_{\gamma}(y) d \mu_{0, \gamma}(y)}, & \text { if } \gamma \in B^{c} \\
0, & \text { if } \gamma \in B,
\end{array}\right.
$$

where $B:=\bar{h}^{-1}(0)$.

Proof. of Theorem E Suppose that $h \in \mathrm{H}_{\zeta}(\Sigma)$. Let $\left(\left\{\mu_{0, \gamma}\right\}_{\gamma}, \phi_{x}\right)$ be the disintegration of $\mu_{0}$ and denote by $\nu$ the measure $\nu:=h \mu_{0}\left(h \mu_{0}(E):=\right.$ $\left.\int_{E} h d \mu_{0}\right)$. If $\left(\left\{\nu_{\gamma}\right\}_{\gamma}, \widehat{\nu}\right)$ is the disintegration of $\nu$, then it holds $\widehat{\nu} \ll m_{1}$ and $\nu_{\gamma} \ll \mu_{0, \gamma}$ (see Lemma 8.1). Moreover, denoting $\bar{h}:=\frac{d \widehat{\nu}}{d m_{1}}$ as (45) and

$$
\frac{d \nu_{\gamma}}{d \mu_{0, \gamma}}(y)=\left\{\begin{array}{cl}
\frac{h(\gamma, y)}{\bar{h}(\gamma)}, & \text { if } \bar{h}(\gamma) \neq 0 \\
0, & \text { if } \bar{h}(\gamma)=0
\end{array}\right.
$$

It is immediate that $\nu \in \mathcal{L}^{\infty}$. Let us check that $\bar{h} \in H_{\zeta}$ by estimating the Hölder constant of $\bar{h}$. For $\gamma_{1}, \gamma_{2} \in M$, we have

$$
\begin{aligned}
\left|\bar{h}\left(\gamma_{2}\right)-\bar{h}\left(\gamma_{1}\right)\right| & \leq\left|\int_{M} h\left(\gamma_{2}, y\right) d\left(\left.\mu_{0}\right|_{\gamma_{2}}\right)-\int_{M} h\left(\gamma_{1}, y\right) d\left(\left.\mu_{0}\right|_{\gamma_{1}}\right)\right| \\
& \leq\left|\int_{M} h\left(\gamma_{2}, y\right) d\left(\left.\mu_{0}\right|_{\gamma_{2}}\right)-\int_{M} h\left(\gamma_{2}, y\right) d\left(\left.\mu_{0}\right|_{\gamma_{1}}\right)\right| \\
& +\left|\int_{M} h\left(\gamma_{2}, y\right) d\left(\left.\mu_{0}\right|_{\gamma_{1}}\right)-\int_{M} h\left(\gamma_{1}, y\right) d\left(\left.\mu_{0}\right|_{\gamma_{1}}\right)\right| \\
& \leq\left|\int_{M} h\left(\gamma_{2}, y\right) d\left(\left.\mu_{0}\right|_{\gamma_{2}}-\left.\mu_{0}\right|_{\gamma_{1}}\right)\right| \\
& +\left|\int_{M} h\left(\gamma_{2}, y\right)-h\left(\gamma_{1}, y\right) d\left(\left.\mu_{0}\right|_{\gamma_{1}}\right)\right| \\
& \leq\left.\left.|| h\right|_{\zeta}|| \mu_{0}\right|_{\gamma_{2}}-\left.\mu_{0}\right|_{\gamma_{1}}||_{W}+H_{\zeta}(h) d_{1}\left(\gamma_{2}, \gamma_{1}\right)^{\zeta}\left|\phi_{x}\right|_{\infty} \\
& \leq\left.|| h\right|_{\zeta}\left|\mu_{0}\right| \zeta d_{1}\left(\gamma_{2}, \gamma_{1}\right)^{\zeta}+H_{\zeta}(h) d_{1}\left(\gamma_{2}, \gamma_{1}\right)^{\zeta}\left|\phi_{x}\right|_{\infty} .
\end{aligned}
$$

Thus, $\bar{h} \in H_{\zeta}$.

\section{Appendix 1: On Disintegration of Measures}

In this section, we prove some results on disintegration of absolutely continuous measures with respect to a measure $\mu_{0} \in \mathcal{A B}$. Precisely, we are going to prove Lemma 8.1. We warn the reader that in this section and the next we work with a slightly different notation.

Let us fix some notations. Denote by $\left(N_{1}, m_{1}\right)$ and $\left(N_{2}, m_{2}\right)$ the spaces defined in section 2.1. For a $\mu_{0}$-integrable function $f: N_{1} \times N_{2} \longrightarrow \mathbb{R}$ and a 
pair $(\gamma, y) \in N_{1} \times N_{2}\left(\gamma \in N_{1}\right.$ and $\left.y \in N_{2}\right)$ we denote by $f_{\gamma}: N_{2} \longrightarrow \mathbb{R}$, the function defined by $f_{\gamma}(y)=f(\gamma, y)$ and $\left.f\right|_{\gamma}$ the restriction of $f$ on the set $\{\gamma\} \times N_{2}$. Then $f_{\gamma}=\left.f\right|_{\gamma} \circ \pi_{y, \gamma}^{-1}$ and $f_{\gamma} \circ \pi_{y, \gamma}=\left.f\right|_{\gamma}$, where $\pi_{y, \gamma}$ is restriction of the projection $\pi_{y}(\gamma, y):=y$ on the set $\{\gamma\} \times N_{2}$. When no confusion is possible, we will denote the leaf $\{\gamma\} \times N_{2}$, just by $\gamma$.

From now on, for a given positive measure $\mu \in \mathcal{A B}$, on $N_{1} \times N_{2}, \widehat{\mu}$ stands for the measure $\pi_{x *} \mu$, where $\pi_{x}$ is the projection on the first coordinate, $\pi_{x}(x, y)=x$.

For each measurable set $A \subset N_{1}$, define $g: N_{1} \longrightarrow \mathbb{R}$ by

$$
g(\gamma)=\left.\left.\phi_{x}(\gamma) \int \chi_{\pi_{x}^{-1}(A)}\right|_{\gamma}(y) f\right|_{\gamma}(y) d \mu_{0, \gamma}(y)
$$

and note that

$$
g(\gamma)= \begin{cases}\left.\phi_{x}(\gamma) \int f\right|_{\gamma}(y) d \mu_{0, \gamma}, & \text { if } \gamma \in A \\ 0, & \text { if } \gamma \notin A\end{cases}
$$

Then, it holds

$$
g(\gamma)=\left.\chi_{A}(\gamma) \phi_{x}(\gamma) \int f\right|_{\gamma}(y) d \mu_{0, \gamma}
$$

Proof. (of Lemma 8.1)

For each measurable set $A \subset N_{1}$, we have

$$
\begin{aligned}
\int_{A} \frac{\pi_{x}^{*}\left(f \mu_{0}\right)}{d m_{1}} d m_{1} & =\int \chi_{A} \circ \pi_{x} d\left(f \mu_{0}\right) \\
& =\int \chi_{\pi_{x}^{-1}(A)} f d \mu_{0} \\
& =\int\left[\left.\left.\int \chi_{\pi_{x}^{-1}(A)}\right|_{\gamma}(y) f\right|_{\gamma}(y) d \mu_{0, \gamma}(y)\right] d\left(\phi_{x} m_{1}\right)(\gamma) \\
& =\int\left[\left.\left.\phi_{x}(\gamma) \int \chi_{\pi_{x}^{-1}(A)}\right|_{\gamma}(y) f\right|_{\gamma}(y) d \mu_{0, \gamma}(y)\right] d\left(m_{1}\right)(\gamma) \\
& =\int g(\gamma) d\left(m_{1}\right)(\gamma) \\
& =\int\left[\left.\int f_{\gamma}(y) d \mu_{0}\right|_{\gamma}(y)\right] d\left(m_{1}\right)(\gamma) .
\end{aligned}
$$

Thus, it holds

$$
\frac{\pi_{x *}\left(f \mu_{0}\right)}{d m_{1}}(\gamma)=\left.\int f_{\gamma}(y) d \mu_{0}\right|_{\gamma}, \text { for } m_{1} \text { - a.e. } \gamma \in N_{1} .
$$

And by a straightforward computation

$$
\frac{\pi_{x *}\left(f \mu_{0}\right)}{d m_{1}}(\gamma)=\left.\phi_{x}(\gamma) \int f\right|_{\gamma}(y) d \mu_{0, \gamma} \text {, for } m_{1}-\text { a.e. } \gamma \in N_{1} \text {. }
$$

Thus, equation (45) is established. 
DECAY OF CORRELATIONS AND HÖLDER REGULARITY FOR SKEW PRODUCT\$1

Remark 9.1. Setting

$$
\bar{f}:=\frac{\pi_{x *}\left(f \mu_{0}\right)}{d m_{1}},
$$

we get, by equation (47), $\bar{f}(\gamma)=0$ iff $\phi_{x}(\gamma)=0$ or $\left.\int f\right|_{\gamma}(y) d \mu_{0, \gamma}(y)=0$, for $m_{1}$-a.e. $\gamma \in N_{1}$.

Now, let us see that, by the $\widehat{\nu}$-uniqueness of the disintegration, equation (46) holds. To do it, define, for $m_{1}$-a.e. $\gamma \in N_{1}$, the function $h_{\gamma}: N_{2} \longrightarrow \mathbb{R}$, in a way that

$$
h_{\gamma}(y)= \begin{cases}\frac{\left.f\right|_{\gamma}(y)}{\left.\int f\right|_{\gamma}(y) d \mu_{0, \gamma}(y)}, & \text { if } \gamma \in B^{c} \\ 0, & \text { if } \gamma \in B .\end{cases}
$$

Let us prove equation (46) by showing that, for all measurable set $E \subset$ $N_{1} \times N_{2}$, it holds

$$
f \mu_{0}(E)=\int_{N_{1}} \int_{E \cap \gamma} h_{\gamma}(y) d \mu_{0, \gamma}(y) d\left(\pi_{x *}\left(f \mu_{0}\right)\right)(\gamma) .
$$

In fact, by equations (47), (48), (9) and remark 9.1, we get

$$
\begin{aligned}
f \mu_{0} & (E)=\int_{E} f d \mu_{0} \\
& =\left.\int_{N_{1}} \int_{E \cap \gamma} f\right|_{\gamma} d \mu_{0, \gamma} d\left(\phi_{x} m_{1}\right)(\gamma) \\
& =\left.\int_{B^{c}} \int_{E \cap \gamma} f\right|_{\gamma} d \mu_{0, \gamma} d\left(\phi_{x} m_{1}\right)(\gamma) \\
& =\left.\int_{B^{c}} \int f\right|_{\gamma}(y) d \mu_{0, \gamma}(y) \phi_{x}(\gamma)\left[\left.\frac{1}{\left.\int f\right|_{\gamma}(y) d \mu_{0, \gamma}(y)} \int_{E \cap \gamma} f\right|_{\gamma} d \mu_{0, \gamma}\right] d m_{1}(\gamma) \\
& =\int_{B^{c}} \bar{f}(\gamma)\left[\left.\frac{1}{\left.\int f\right|_{\gamma}(y) d \mu_{0, \gamma}(y)} \int_{E \cap \gamma} f\right|_{\gamma} d \mu_{0, \gamma}\right] d m_{1}(\gamma) \\
& =\int_{B^{c}}\left[\left.\frac{1}{\left.\int f\right|_{\gamma}(y) d \mu_{0, \gamma}(y)} \int_{E \cap \gamma} f\right|_{\gamma} d \mu_{0, \gamma}\right] d \bar{f} m_{1}(\gamma) \\
& =\int_{B^{c}} \int_{E \cap \gamma} h_{\gamma}(y) d \mu_{0, \gamma}(y) d\left(\pi_{x *}\left(f \mu_{0}\right)\right)(\gamma) \\
= & \int_{N_{1}} \int_{E \cap \gamma} h_{\gamma}(y) d \mu_{0, \gamma}(y) d\left(\pi_{x *}\left(f \mu_{0}\right)\right)(\gamma) .
\end{aligned}
$$

And we are done.

\section{REFERENCES}

[1] [10.4064/fm224-3-2] J. F. Alves and M. Soufi, Statistical stability of geometric Lorenz attractors, Fundamenta Mathematicae, 224 (2014), 219-231. 
[2] [10.1007/s00209-013-1231-0] V. Araujo, S. Galatolo and M. Pacifico, Decay of correlations for maps with uniformily contracting fibers and logarithm law for singular hyperbolic attractors, Mathematische Zeitschrift, 276 (2012).

[3] [10.1007/978-3-642-11414-4] V. Araujo and M. Pacifico, Three-dimensional flows, Springer-Verlag, New York, 2010.

[4] [10.1017/etds.2018.28] W. Bahsoun and M. Ruziboev, On the statistical stability of Lorenz attractors with a $C^{1+\alpha}$ stable foliation, Ergodic Theory and Dynamical Systems, 39 (2019), 3169-3184.

[5] [10.1007/s10955-016-1663-0] V. Baladi, The quest for the ultimate anisotropic Banach space, Journal of Statistical Physics, 166 (2016), 525-557.

[6] [10.1142/9789812813633] V. Baladi, Positive transfer operators and decay of correlations, World Scientific Pub Co Inc, London, 2000.

[7] [10.3934/jmd.2010.4.91] V. Baladi and S. Gouëzel, Banach spaces for piecewise cone hyperbolic maps, Journal of Modern Dynamics, 4 (2009), 91-137.

[8] [10.5802/aif.2253] V. Baladi and M. Tsujii, Anisotropic Hölder and Sobolev spaces for hyperbolic diffeomorphisms, Annales de l'institut Fourier, 57 (2007), 127-154.

[9] [10.1016/j.anihpc.2009.01.001] V. Baladi and S. Gouëzel, Good Banach spaces for piecewise hyperbolic maps via interpolation, Annales de l'Institut Henri Poincare (C) Non Linear Analysis, 26 (2007), 1453-1481.

[10] [10.1007/978-1-4612-2024-4] A. Boyarsky and P. Gora, Laws of Chaos - Invariant Measures and Dynamical Systems in One Dimension, Birkhauser, Boston, 1997.

[11] [10.3934/jmd.2007.1.301] O. Butterley and C. Liverani, Smooth Anosov flows: correlation spectra and stability, J. Mod. Dyn., 1 (2007), 301-322.

[12] [10.1007/s11856-017-1477-z] O. Butterley and I. Melbourne, Disintegration of Invariant Measures for Hyperbolic Skew Products, Isr. J. Math, 219 (2017), 171-188

[13] [10.1017/etds.2015.86] A. Castro and T. Nascimento, Statistical Properties of the maximal entropy measure for partially hyperbolic attractors, Ergodic Theory and Dynamical Systems, 37 (2017), 1060-1101.

[14] [10.1016/j.anihpc.2012.07.004] A. Castro and P. Varandas, Equilibrium states for non-uniformly expanding maps: Decay of correlations and strong stability, Annales de l'Institut Henri Poincare (C) Non Linear Analysis, 30 (2013), 225-249.

[15] [10.1016/j.chaos.2018.08.028] M. Demers, A gentle introduction to anisotropic banach spaces, Chaos, Solitons \& Fractals, 116 (2018), 29-42.

[16] [10.1090/S0002-9947-08-04464-4] M. Demers and C Liverani, Stability of Statistical Properties in Two-Dimensional Piecewise Hyperbolic Maps, Transactions of the American Mathematical Society, 360 (2006), 4777-4814.

[17] [10.3934/jmd.2011.5.665] M. Demers and HZ. Zhang, Spectral analysis of the transfer operator for the Lorentz gas, Journal of Modern Dynamics, 5 (2011), 665-709.

[18] [10.1007/s00220-013-1820-0] M. Demers and HZ. Zhang, A functional Analytic approach to perturbations of the Lorentz gas, Communications in Mathematical Physics, 324 (2013), 767-830.

[19] S. Galatolo, Statistical properties of dynamics. Introduction to the functional analytic approach. Lecture notes for the Hokkaido-Pisa University summer school 2015, preprint, arXiv1510.02615

[20] [10.5802/jep.73] S. Galatolo, Quantitative statistical stability, speed of convergence to equilibrium for partially hyperbolic skew products, Journal de l'cole polytechnique, 5 (2018), 377-405.

[21] [10.3934/dcds.2020079] S. Galatolo and R. Lucena, Spectral Gap and quantitative statistical stability for systems with contracting fibers and Lorenz like maps, Discrete \& Continuous Dynamical Systems - A, 40 (3) (2020), 1309-1360.

[22] [10.3934/jcd.2015.2.51] S. Galatolo, I. Nisoli and B. Saussol, An elementary way to rigorously estimate convergence to equilibrium and escape rates, Journal of Computational Dynamics, 2 (2014), 51-64. 
[23] [10.1017/S0143385709000856] S. Galatolo and M. J. Pacifico, Lorenz-like flows: Exponential decay of correlations for the Poincaré map, logarithm law, quantitative recurrence, Ergodic Theory and Dynamical Systems, 30 (2010), 1703-1737.

[24] [10.1017/S0143385705000374] S. Gouezel and C. Liverani, Banach spaces adapted to Anosov systems, Ergodic Theory and Dynamical Systems, 26 (2006), 189-217.

[25] [10.2307/1969514] C. Ionescu-Tulcea and G. Marinescu, Theorie ergodique pour des classes d' operateurs non completement continues, Annals of Mathematics, 52 (1950), $140-147$.

[26] [10.1007/BF00532744] G. Keller, Generalized bounded variation and applications to piecewise monotonic transformations, Probability Theory and Related Fields, 69 (1985), 461-478.

[27] G. Keller and C. Liverani, Stability of the spectrum for transfer operators, Annali della Scuola Normale Superiore di Pisa. Classe di Scienze. Serie IV, 28 (1999), 141152.

[28] [10.1017/etds.2020.22] B. R. Kloeckner, Extensions with shrinking fibers, Ergodic Theory and Dynamical Systems, (2020), 1-40.

[29] [10.2307/1996575] A. Lasota and J.Yorke, On the Existence of Invariant Measures for Piecewise Monotonic Transformations, Transactions of The American Mathematical Society, 186 (1973), 481-488.

[30] D. Lima and R. Lucena, Lipschitz regularity of the invariant measure and statistical properties for a class of random dynamical systems, https://arxiv.org/abs/2001.08265

[31] [10.1007/BF02183704] C. Liverani, Decay of correlations for piecewise expanding maps, Journal of Statistical Physics, 78 (1995), 1111-1129.

[32] C. Liverani, Invariant measures and their properties. A functional analytic point of view, Scuola Normale Superiore in Pisa, (2004).

[33] [10.2307/2118636] C. Liverani, Decay Of Correlations, Annals of Mathematics, 142 (1997), 239-301.

[34] R. Lucena, Spectral Gap for Contracting Fiber Systems and Applications, Ph.D thesis, Universidade Federal do Rio de Janeiro in Brazil, 2015.

[35] K. Oliveira and M. Viana, Fudamentos da Teoria Ergódica, Colecão Fronteiras da Matematica - SBM, Brazil, 2014.

[36] [10.1214/aop/1176993522] J. Rousseau-Egele, Un Theoreme de la Limite Locale Pour une Classe de Transformations Dilatantes et Monotones par Morceaux, The Annals of Probability, 11 (1983), 772-788.

[37] [10.1016/j.anihpc.2009.10.002] P. Varandas and M. Viana, Existence, uniqueness and stability of equilibrium states for non-uniformly expanding maps, Annales de l'Institut Henri Poincare (C) Non Linear Analysis, 27 (2010), 555-593.

[38] M. Viana, Stochastic dynamics of deterministic systems, Brazillian Math. Colloquium 1997, IMPA http://w3.impa.br/ viana/out/sdds.pdf

(Rafael A. Bilbao) Universidad Pedagógica y Tecnológica de Colombia, Avenida Central del Norte 39-115, Sede Central Tunja, Boyacá, 150003, Colombia.

E-mail address: rafael.alvarez@uptc.edu.co

(Ricardo Bioni) Rua Costa Bastos, 34, Santa Teresa, Rio de Janeiro-Brasil

E-mail address: ricardo.bioni@hotmail.com

(Rafael Lucena) Universidade Federal de Alagoas - UFAL, Av. Lourival Melo

Mota, S/N Tabuleiro do Martins, Maceio - AL, 57072-900, Brasil

E-mail address: rafael.lucena@im.ufal.br

$U R L$ : www.im.ufal.br/professor/rafaellucena 